\title{
Regularity of sets with constant horizontal normal in the Engel group
}

\author{
Costante Bellettini And EnRico Le Donne
}

\begin{abstract}
In the Engel group with its Carnot group structure, we study subsets of locally finite subRiemannian perimeter and possessing constant subRiemannian normal. We prove the rectifiability of such sets: more precisely we show that, in some specific coordinates, they are upper-graphs of entire Lipschitz functions (with respect to the Euclidean distance). However we find that, when they are written as intrinsic horizontal upper-graphs with respect to the direction of the normal, then the function defining the set might even fail to be continuous. Nevertheless, we can prove that one can always find other horizontal directions for which the set is the intrinsic horizontal upper-graph of a function that is Lipschitz-continuous with respect to the intrinsic subRiemannian cones (and in particular locally Hölder-continuous for the Euclidean distance). We further discuss a partial differential equation characterization of the class of all sets with constant horizontal normal. Finally, we show that our rectifiability argument extends to the case of filiform groups of the first kind.
\end{abstract}

\section{Introduction}

Recent years have witnessed an increasing interest in Geometric Analysis of Metric Spaces. A particular role has been played by the class of Carnot groups endowed with subRiemannian distances. In this setting, both translations and dilations are present, hence the theory of differentiation generalizes. Many notions from Analysis and Geometry have been investigated in subRiemannian Carnot groups. Function Theory has been a fruitful study. There have been several fundamental results in the study of maps such as Lipschitz, Sobolev, quasiconformal, and bounded variation. Another subject of large interest has been Geometric Measure Theory. Minimal surfaces, sets with finite perimeter, currents, and rectifiable sets have received particular attention. As a source of reference, we point out to [8, 20, 25], and references therein. 
A theorem of basic importance in the study of Euclidean sets of finite perimeter is that reduced boundaries admit a unique tangent space almost everywhere, in other words they are rectifiable. The tangent is obtained via a blow-up analysis: dilating the set about almost any point on the reduced boundary, one obtains in the limit a uniquely defined half-space [10-12]). The analogous analysis in the subRiemannian setting only yields that the limiting object (after a blow up has been performed) must be a set with constant horizontal normal. The analysis of sets with constant horizontal normal in Carnot groups becomes consequently a matter of primary importance.

Let us shortly describe the state of the art. In Carnot groups of step two, the work of Franchi et al. [14] reveals that such sets are precisely vertical half-spaces. This result has played a key role both in the subsequent development of geometric measure theory in Carnot groups as well as in more far-flung applications, for example the work by Cheeger and Kleiner [9] on the biLipschitz non-embeddability of the Heisenberg group into $L^{1}$. Already in [14], however the authors observed the existence, in step three Carnot groups, of sets with constant horizontal normal that fail to be half-spaces. The complete understanding of sets with constant horizontal normal turns out to be rather hard in full generality and in the present work we aim to give a contribution in this direction by focusing on the simplest Carnot group of step three, the so-called Engel group. The focus on the Engel group is justified by the fact that it allows us to see already the difficulties arising in groups of step at least three, but at the same time the controlled algebraic complexity of the Lie algebra allows us to state precise results on the structure of sets with constant horizontal normal.

After the understanding of sets with constant horizontal normal has become deeper, it is conceivable to go back to the blow-up analysis of sets of finite perimeter, where it is known that any limit must be a set with constant horizontal normal, and face the further-reaching investigation: is any such limit uniquely determined? Remark that, if it is so, then it must be a halfspace by the result of [2]. In the uniqueness issue, the precise knowledge of what possible tangents can arise can play an important role.

The importance of sets with constant horizontal normal goes beyond the fact that they appear as tangents of sets with locally-finite horizontal perimeter. Indeed, their boundaries are examples of minimal hyper-surfaces that can be written as entire graphs with respect to the group structure. Such parameterizations are called horizontal intrinsic graphs. Their importance in the theory of rectifiable subsets of Carnot groups has been addressed in $[3-7,15,16,19,22]$, where they have been extensively studied. 
In step higher than 2, no regularity result is known for sets of constant horizontal normal. Actually, no Euclidean rectifiability is expected. It is not clear whether an intrinsic-rectifiability result can be expected to hold for sets with constant horizontal normal in general Carnot groups. Nor we have examples of finite-perimeter sets for which it fails that, at almost every point of the boundary, the tangent is a half-space.

The aim of this paper is to show that in low step (namely, step 3) we have an intermediate situation. We are going to focus on the lower-dimensional Carnot group of step 3, the Engel group, and provide a collection of results that address the problem of regularity for sets with constant horizontal normal from several points of view.

We shall show a structural geometric property, to wit, any such set is Lebesgue equivalent to a Euclidean Lipschitz domain. In particular, such sets are rectifiable (even in the Euclidean sense).

We shall then give a more concrete and precise regularity result by using the model of the Engel group corresponding to the so-called exponential coordinates of the second kind. Namely, in this model we are able to: (i) describe any set with constant horizontal normal as the upper-graph of an entire Lipschitz function and (ii) reformulate the constant horizontal normal condition as a distributional partial differential inequality.

We shall analyse the problem of whether it is possible to express any given set with constant horizontal normal as an intrinsic horizontal uppergraph with respect to specific horizontal vectors and what regularity to expect for such a graph. In fact, there are examples of sets with constant horizontal normal such that, if one writes the set as an intrinsic horizontal upper-graph in the direction of the normal, the function giving the graph is not even continuous. However, all constant normal sets are intrinsic Lipschitz upper-graphs in other horizontal directions. This last feature is peculiar to the Engel group. In a subsequent work we prove that in general Carnot groups sets with constant horizontal normal might fail to be intrinsic Lipschitz upper-graphs in every horizontal direction.

\subsection{Main setting, terminology, and previous contributions}

Let $\mathbb{G}$ be a Carnot group (see [2] for definitions). Let $\mathfrak{g}$ be the Lie algebra of the left-invariant vector fields in $\mathbb{G}$. By definition, $\mathfrak{g}$ is stratified. We denote by $V_{1}$ the first stratum (also known as horizontal layer).

A subset $E$ of a Carnot group is said to have locally finite horizontal perimeter if, for any $X \in V_{1}$, the distribution $X \mathbb{1}_{E}$ is a Radon measure. Caccioppoli and De Giorgi introduced these sets (in the Euclidean space) 
for the study of minimal hyper-surfaces. The reason for doing so is the good behaviour of the perimeter, which is the total mass of the vectorvalued measure whose components are obtained by differentiating $\mathbb{1}_{E}$ in the directions of a fixed basis of $V_{1}$. In fact, the perimeter is lower semicontinuous and induces a locally compact topology on the class of finite-perimeter sets. Hence, it becomes easy to show existence of minimal surfaces.

A set $E$ in a Carnot group $\mathbb{G}$ is said to have constant horizontal normal if there exists a horizontal left-invariant vector field $X$ on $\mathbb{G}$ and there exists a decomposition $\mathbb{R} X \oplus V_{1}^{\dagger}$ of $V_{1}$ with the following property:

- the distributional derivative $X \mathbb{1}_{E}$ of the characteristic function $\mathbb{1}_{E}$ of $E$ in the direction of $X$ is a positive Radon measure;

- for all $Y \in V_{1}^{\dagger}$, the distribution $Y \mathbb{1}_{E}$ vanishes.

One should notice that the space $V_{1}^{\dagger}$ is uniquely defined by $E$, unlike the vector $X$. However, if we fix a scalar product on $V_{1}$ and require that $X$ is a unit vector orthogonal to $V_{1}^{\dagger}$, then $X$ is unique and it is called the normal of $E$.

In [14], the three authors extended a result of De Giorgi by proving rectifiability of sets with locally finite horizontal perimeter in Carnot groups of step 2. Following De Giorgi's strategy, they obtained this result, by showing that almost every tangent is a set of constant normal and that constant-normal sets are in fact half-spaces. Alas, they noticed that this latter fact does not hold in higher-step Carnot groups. In fact, in [14, Example 3.2], they gave the first explicit example of a subset of the Engel group with constant normal that is not a half-space. More examples have then been given in [2] and we will be adding some more in the present work.

In [4], the three authors showed that sets with constant horizontal normal are calibrated sets: the calibration that they used is the scalar product with the normal. This calibration method implies that, in any Carnot group, boundaries of sets with constant horizontal normal are minimal surfaces, just as it happens in the Euclidean framework. Guided by the Euclidean experience, one could expect fairly good results on the smoothness of calibrated sets: the classical regularity theory for minimal sets in $\mathbb{R}^{n}$ indeed argues, in its key steps, as follows. First write the set, locally around a point where we have a tangent plane, as a graph on the tangent plane; then prove that the normal is Hölder continuous. It is now crucial the fact that, in this Euclidean setting, one can further improve regularity to $C^{1}$ (for the original proofs see $[12,17])$. 
Going back to the subRiemannian framework, one can write any constant-normal set as an intrinsic upper-graph in the direction of the normal. Such graphs have been considered in $[3-7,15,16,19,22]$ and give canonical parameterizations. The result of [23] suggested that sets that are uppergraphs of functions with controlled normal (e.g., constant) should admit some regularity. This behaviour occurs indeed in the Euclidean case, as previously indicated. We shall give examples in the Engel group of sets with constant horizontal normal where the function is not even continuous. Here the choice of a specific normal direction (namely, of a scalar product on $V_{1}$ ) will be crucial; indeed we can on the other hand prove that, by choosing other horizontal directions, we can express the set as upper-graph of a function taking values in the new direction, and this function is intrinsically Lipschitz continuous, i.e., Lipschitz with respect to cones in the intrinsic subRiemannian geometry, in particular it is locally Euclidean Hölder continuous.

\subsection{Overview of results}

We recall now the definition of the Carnot group of interest to us, the Engel group. The Engel algebra is the Lie algebra generated, as vector space, by four vectors $X_{1}, X_{2}, X_{3}, X_{4}$, with relations

$$
\begin{gathered}
{\left[X_{1}, X_{2}\right]=X_{3} \quad \text { and } \quad\left[X_{1}, X_{3}\right]=X_{4},} \\
{\left[X_{1}, X_{4}\right]=\left[X_{2}, X_{4}\right]=\left[X_{2}, X_{3}\right]=\left[X_{3}, X_{4}\right]=0}
\end{gathered}
$$

Such an algebra is nilpotent of step 3 and stratified by the strata

$$
V_{1}:=\mathbb{R} X_{1} \oplus \mathbb{R} X_{2}, \quad V_{2}:=\mathbb{R} X_{3}, \quad V_{3}:=\mathbb{R} X_{4}
$$

The Engel group is defined as the unique connected and simply connected Lie group with the Engel algebra as Lie algebra. Through the paper we denote by $\mathbb{G}$ such a group.

We endow the Engel group $\mathbb{G}$ with some Haar measure $\operatorname{vol}_{\mathbb{G}}$. Given a measurable set $E \subset \mathbb{G}$ and a left-invariant vector field $X \in \operatorname{Lie}(G)$, we write

$$
X \mathbb{1}_{E} \geq 0 \quad\left(\operatorname{resp} . X \mathbb{1}_{E}=0\right)
$$

if, for all $\phi \in C_{c}^{\infty}(\mathbb{G})$ with $\phi \geq 0$,

$$
-\int_{E} X \phi d \operatorname{vol}_{\mathbb{G}} \geq 0 \quad\left(\operatorname{resp} . \int_{E} X \phi d \operatorname{vol}_{\mathbb{G}}=0\right) .
$$


Since the flow of a left-invariant vector field is a right translation, then the flow of such a vector field preserves the Haar measure, which on a nilpotent group is always biinvariant. In other words, any element of the Lie algebra is a divergence-free vector field on the manifold $\mathbb{G}$, endowed with a Haar measure $\operatorname{vol}_{\mathbb{G}}$.

Definition 1.2 (Constant horizontal normal). Let $V_{1}$ be the first stratum of Lie $(\mathbb{G})$. Fix a scalar product $\langle\cdot \mid \cdot\rangle$ on $V_{1}$. A set $E \subseteq \mathbb{G}$ is said to have constant horizontal normal $X \in \operatorname{Lie}(\mathbb{G})$ if $X \in V_{1}, X \mathbb{1}_{E} \geq 0$, and

$$
Y \in V_{1}, \quad\langle X \mid Y\rangle=0 \Longrightarrow Y \mathbb{1}_{E}=0 .
$$

A direction $X$ such that $X \mathbb{1}_{E} \geq 0$ will be referred to as a monotone direction for the set $E$.

Regarding the next definition, recall that, being connected, simply connected, and nilpotent, the Engel group $\mathbb{G}$ is diffeomorphic to Lie $(\mathbb{G})$, via the exponential map, and so is diffeomorphic to $\mathbb{R}^{4}$.

Definition 1.3 (Euclidean Lipschitz domain). A set $E \subseteq \mathbb{G}$ is called an Euclidean Lipschitz domain if, for one (and thus for all) diffeomorphisms $f: \mathbb{G} \rightarrow \mathbb{R}^{4}$, the set $f(E)$ is a Lipschitz domain of $\mathbb{R}^{4}$. Namely, $f(E)$ is an open set and any point on the boundary has a neighbourhood in which the set can be described as the upper-graph of a Lipschitz map of three variables.

Our first result is the following.

Theorem 1.4. If $E$ is a subset of the Engel group $\mathbb{G}$ that has constant horizontal normal, then there exists an Euclidean Lipschitz domain $\tilde{E} \subset \mathbb{G}$ that is equivalent to $E$, i.e., it is such that $\operatorname{vol}(E \Delta \tilde{E})=0$. In the particular case that the normal is $X_{1}$, then $E$ is equivalent more precisely to a vertical half-space.

We give now a sketch of the proof, referring for the intermediate steps to subsequent subsections of the paper. Let $Y_{1}=X$ be the normal of $E$. Take $Y_{2} \in V_{1}$ with $\left\langle X \mid Y_{2}\right\rangle=0$. Hence $Y_{1} \mathbb{1}_{E} \geq 0$ and $Y_{2} \mathbb{1}_{E}=0$. A result of [2, Proposition 4.7] allows us to obtain two extra monotone directions for $E$, namely we get (see Section 2.1) $Y_{3}, Y_{4} \in \operatorname{Lie}(\mathbb{G})$ such that $Y_{1}, Y_{2}, Y_{3}, Y_{4}$ form a basis, $Y_{3} \mathbb{1}_{E} \geq 0$, and $Y_{4} \mathbb{1}_{E} \geq 0$. Take the Lebesgue representative $\tilde{E}$ of $E$ (Section 2.2). The set $\tilde{E}$ will have the property that, for all $p \in \partial \tilde{E}$ and 
for all $Z=\sum_{j=1}^{4} \alpha_{j} Y_{j}$ with $\alpha_{j}>0$, one has that

$$
\{t \in \mathbb{R}: p \exp (t Z) \in \tilde{E}\}=(0,+\infty)
$$

In other words, the "cone"

$$
C_{p}:=\left\{p \exp \left(\sum_{j=1}^{4} \alpha_{j} Y_{j}\right): \alpha_{j}>0\right\}
$$

does not intersect $\tilde{E}$. Finally, a standard cone criterion gives the Lipschitz regularity of $\partial \tilde{E}$.

We remark that Theorem 1.4 is an intrinsic structural statement for $E$, i.e., it only depends on the differentiable structure of $\mathbb{G}$ and not on the specific choice of a coordinate system. However, from the proof just sketched we shall draw a more precise characterization of any set with constant horizontal normal when we use the model of the Engel group corresponding to the use of exponential coordinates of the second kind (see the beginning of Section 3.1 for the definition). Observe that the cone $C_{p}$ is a left translation of $C_{q}$, for $p, q \in \partial \tilde{E}$. We show that in exponential coordinates of second kind there exists an Euclidean cone $C$ such that the set $C_{p}$ contains the translated cone $p+C$. Thus we conclude that $\partial \tilde{E}$ is an entire Lipschitz graph:

Theorem 1.5. Let $\mathbb{G}$ be the Engel group. Let $\Psi: \mathbb{R}^{4} \rightarrow \mathbb{G}$ be the exponential coordinates of the second kind. For all horizontal vector $X$, exists a basis $w_{1}, \ldots, w_{4} \in \mathbb{R}^{4}$ with the following property. If $E \subset \mathbb{G}$ is any subset that has constant horizontal normal $X$, then there exists a 1-Lipschitz map $h: \mathbb{R}^{3} \rightarrow$ $\mathbb{R}$ such that $E$ is equivalent to

$$
\left\{\Psi\left(a_{1} w_{1}+\cdots+a_{4} w_{4}\right): a_{1}, a_{2}, a_{3} \in \mathbb{R}, a_{4}>h\left(a_{1}, a_{2}, a_{3}\right)\right\}
$$

The rectifiability argument of Theorem 1.4 can be extended almost verbatim to all "filiform groups of the first kind". See Section 5 for a discussion on filiform group and the proof of the result.

To complete the description of sets with constant horizontal normal in the Engel group in exponential coordinates of second kind we shall give an analytic characterization of any such set as upper-graph of a function that satisfies a partial differential inequality. Recall from Theorem 1.4 that the 
only non-trivial case to address is the one of constant normal different from $X_{1}$. Roughly speaking we show:

Theorem (See Theorem 3.17). For any $X \neq X_{1}$ there is a suitable choice of exponential coordinates $\left(x_{1}, x_{2}, x_{3}, x_{4}\right)$ of the second kind such that the following holds. A set has finite perimeter and constant horizontal normal $X$ if and only if it is of the form $\left\{x_{2}>G\left(x_{3}, x_{4}\right)\right\}$ for a $B V$ function $G: \mathbb{R}^{2} \rightarrow \overline{\mathbb{R}}=\{-\infty\} \cup \mathbb{R} \cup\{+\infty\}$ that satisfies the following partial differential inequality: for all $h \in C_{c}^{\infty}(\mathbb{G})$ such that $h \geq 0$, it holds

$$
\left(\left\langle\partial_{3} G, h\right\rangle\right)^{2}+2\left\langle\partial_{4} G, h\right\rangle\left\langle\mathcal{L}^{2}, h\right\rangle \leq 0
$$

Here $\mathcal{L}^{2}$ denotes the Lebesgue measure on $\mathbb{R}^{2}$ and $\langle$,$\rangle denotes the pairing of$ distributions and smooth test functions.

The statement of the result requires a suitable notion of BV-function taking values into the extended real line $\overline{\mathbb{R}}$ and would be a bit too technical for this introduction: the precise features of $G$ and the meaning of the partial differential inequality (1.6) are therefore postponed to Theorem 3.17. Minimal graphs of functions that also assume the values $+\infty$ and $-\infty$ have already appeared in the Euclidean setting. For example, Miranda considered them in the solution of the Dirichlet's problem for the minimal surfaces equation; see [21] and [17, Chapter 16].

It should be noted that the 'Euclidean Lipschitz continuity' obtained in Theorem 1.5, as well as the analytic characterization just given, require the expression of $E$ as an upper-graph with respect to a non-horizontal direction. We thus now turn our attention to the expression of $E$ as an "algebraically intrinsic horizontal graph" (see Section 4). This means that we express $E$ as union of half-flow-lines in a horizontal left-invariant direction. More precisely:

Definition 1.7 (Intrinsic horizontal upper-graph). Given a Carnot group $\mathbb{G}$, let $X \in \operatorname{Lie}(G)$ be a horizontal direction. Let $W$ be a subgroup that is complementary to $\exp (\mathbb{R} X)$. Let $T: W \rightarrow \mathbb{R}$ be any function. We say that a set $E \subset \mathbb{G}$ is an intrinsic horizontal upper-graph in the direction $X$ if

$$
E=\{w \exp (t X): w \in W, t>T(w)\}
$$

One should observe that the point $w \exp (t X)$ is the flow from $w$ for time $t$ in the direction $X$. We will express any set with constant horizontal 
normal $E$ as an intrinsic horizontal upper-graph as in (1.8). Understanding the regularity of $T$ is then our next task.

The most natural horizontal direction to use would seem to be the direction of the normal. Nevertheless we will find, in Section 4:

Theorem (See Theorem 4.1). For any $X \neq X_{1}$, we can provide an example of subset $E \subset \mathbb{G}$ with constant horizontal normal $X$ and with the property that, when $E$ is expressed as intrinsic horizontal upper-graph in the direction of $X$, the function for which it is upper-graph is not continuous.

Theorem (See Theorem 4.6). Let $E \subset \mathbb{G}$ be an arbitrary set having constant horizontal normal $X$. Whenever $E$ is written as intrinsic horizontal upper-graph using a horizontal direction $Y$ with $\langle X, Y\rangle \neq 0$ and $X$ not parallel to $Y$, then the function for which it is upper-graph is Lipschitz continuous with respect to intrinsic cones in $\mathbb{G}$ (see Definition 4.5). In particular, the function is locally Hölder continuous for the Euclidean distance.

Such intrinsic cones are cones with respect to the intrinsic subRiemannian geometry. The above result confirms that the natural notion (introduced by Franchi et al. in [15], see also [16]) of intrinsic Lipschitz continuity to be used for intrinsic horizontal graphs in the subRiemannian context is the one with respect to the intrinsic Carnot-Caratheodory distance.

\section{Proof of Theorem 1.4}

\subsection{Getting more monotone directions}

Let $E$ be a subset of the Engel group $\mathbb{G}$ that has constant horizontal normal $X \in \operatorname{Lie}(\mathbb{G})$. Let $Y$ be a vector in $V_{1}$ that is orthogonal to $X$. Note that the line $\mathbb{R} Y$ is independent from the scalar product chosen on $V_{1}$. Now we face a dichotomy: either $Y$ is parallel to the vector $X_{2}$ of the definition of the Lie algebra representation $(1.1)$ of $\operatorname{Lie}(\mathbb{G})$, or not. In the second case, we show that we can change the basis of $\operatorname{Lie}(\mathbb{G})$ and assume that $Y=X_{1}$.

The case $Y=X_{2}$ is easy to handle and in fact we show that $E$ is (equivalent to) a half-space. In the case $Y=X_{1}$ instead, it is not necessarily true that $E$ is a half-space. Examples to this effect were first given in [14].

In both cases, we are going to make use of the following property of stability of monotone directions.

Proposition 2.1 (see [2, Proposition 4.7]). Let $X, Y \in \operatorname{Lie}(\mathbb{G})$ and $E \subset \mathbb{G}$. Assume that $X \mathbb{1}_{E}=0$ and $Y \mathbb{1}_{E} \geq 0$. Then $\left(\operatorname{Ad}_{\exp (X)} Y\right) \mathbb{1}_{E} \geq 0$. 
Recall that

$$
\operatorname{Ad}_{\exp (X)} Y=e^{\operatorname{ad}_{X}} Y=Y+[X, Y]+\frac{1}{2}[X,[X, Y]]
$$

in a 3-step group.

Easy case: sets with normal $X_{1}$.

Let $\mathbb{G}$ be the Engel group whose Lie algebra is generated by $X_{1}, X_{2}, X_{3}$, $X_{4}$ with relations $(1.1)$.

Lemma 2.2. Let $E \subset \mathbb{G}$. Assume that $X_{1} \mathbb{1}_{E} \geq 0$ and $X_{2} \mathbb{1}_{E}=0$. Then $E$ is a vertical half-space.

For the definition and other characterization of half-spaces see [2].

Proof of Lemma 2.2. Applying Proposition 2.1 with $X=t X_{2}, Y=X_{1}$, and $t \in \mathbb{R}$, we get that the vector field

$$
Z:=\operatorname{Ad}_{\exp \left(t X_{2}\right)} X_{1}=X_{1}+\left[t X_{2}, X_{1}\right]+\frac{1}{2}\left[t X_{2},\left[t X_{2}, X_{1}\right]\right]=X_{1}-t X_{3}
$$

is such that $Z \mathbb{1}_{E} \geq 0$, for all $t \in \mathbb{R}$. Letting $t \rightarrow+\infty$ and $t \rightarrow-\infty$, respectively, we get that both $-X_{3} \mathbb{1}_{E} \geq 0$ and $X_{3} \mathbb{1}_{E} \geq 0$. Hence $X_{3} \mathbb{1}_{E}=0$. Apply again the proposition with $X=t X_{3}, Y=X_{1}$, and $t \in \mathbb{R}$. Thus, the vector

$$
Z^{\prime}:=\operatorname{Ad}_{\exp \left(t X_{3}\right)} X_{1}=X_{1}+\left[t X_{3}, X_{1}\right]=X_{1}-t X_{4}
$$

is such that $Z^{\prime} \mathbb{1}_{E} \geq 0$, for all $t \in \mathbb{R}$. Arguing as before, we conclude that $X_{4} \mathbb{1}_{E}=0$. By the BV characterization of vertical half-spaces, see [2, Proposition 4.4], we are done.

Hard case: sets with normal $X_{2}$.

We first argue that if the normal is not $X_{1}$ then we can assume that the normal is $X_{2}$.

Lemma 2.3. Let $\mathfrak{g}$ be the Engel algebra with basis $X_{1}, X_{2}, X_{3}, X_{4}$ and relations (1.1). Let $X=\alpha X_{1}+\beta X_{2}$ with $\alpha, \beta \in \mathbb{R}$ and $\alpha \neq 0$. Then there exists a Lie algebra strata-preserving endomorphism $\psi$ of $\mathfrak{g}$ such that $\psi X_{1}=X$. 
Proof. Define $\psi: \mathfrak{g} \rightarrow \mathfrak{g}$ by the property

$$
\psi X_{1}=\alpha X_{1}+\beta X_{2} \text { and } \psi X_{2}=X_{2},
$$

and

$$
\psi X_{3}=\alpha X_{3} \quad \text { and } \quad \psi X_{4}=\alpha^{2} X_{4} .
$$

It is straightforward ${ }^{1}$ to check that such a $\psi$ is an isomorphism.

By the above lemma, the following fact is immediate.

Corollary 2.4. Let $E$ be a subset of the Engel group $\mathbb{G}$. Let $X, Y \in V_{1} \subset$ $\operatorname{Lie}(\mathbb{G})$ linearly independent. Assume $X \mathbb{1}_{E} \geq 0$ and $Y \mathbb{1}_{E}=0$. Then there exists a basis $X_{1}, X_{2}, X_{3}, X_{4}$ of $\operatorname{Lie}(\mathbb{G})$ with relations (1.1) such that

- either $X_{1} \mathbb{1}_{E} \geq 0$ and $X_{2} \mathbb{1}_{E}=0$,

- or $X_{1} \mathbb{1}_{E}=0$ and $X_{2} \mathbb{1}_{E} \geq 0$.

In other words, we only need to study the cases where either $E$ has normal $X_{1}$ or it has normal $X_{2}$. Since we already solved the first case, let us focus now on the second.

If one applies Proposition 2.1 to the case of constant normal equal to $X_{2}$, one obtains the following.

Lemma 2.5. Let $E \subset \mathbb{G}$. Assume that $X_{2} \mathbb{1}_{E} \geq 0$ and $X_{1} \mathbb{1}_{E}=0$. Then, for all $t \in \mathbb{R}$, the vector

$$
Z_{t}:=X_{2}+t X_{3}+\frac{t^{2}}{2} X_{4}
$$

is such that $Z_{t} \mathbb{1}_{E} \geq 0$. In particular,

$$
X_{4} \mathbb{1}_{E} \geq 0 \quad \text { and } \quad\left(X_{2}+2 X_{3}+2 X_{4}\right) \mathbb{1}_{E} \geq 0 .
$$

\subsection{Sets with constant horizontal normal are Euclidean Lipschitz domains}

In this section we will prove Theorem 1.4 by showing that the Lebesgue representative $\tilde{E}$ of a set $E$ having constant horizontal normal $X_{2}$ satisfies a "cone criterion" and is therefore an Euclidean Lipschitz domain. Recall that we have already shown that the case of constant horizontal normal $X_{1}$ is

\footnotetext{
${ }^{1}$ Here is the calculation: $\psi\left[X_{1}, X_{2}\right]=\psi X_{3}=\alpha X_{3}=\alpha\left[X_{1}, X_{2}\right]=\left[\psi X_{1}, \psi X_{2}\right]$ and $\psi\left[X_{1}, X_{3}\right]=\psi X_{4}=\alpha^{2} X_{4}=\alpha^{2}\left[X_{1}, X_{3}\right]=\left[\psi X_{1}, \psi X_{3}\right]$.
} 
easy to handle (Lemma 2.2), so we only need to focus on the case of normal $X_{2}$ (by Lemma 2.3).

One should note at this point that the four vectors

$$
Y_{1}:=X_{1}, \quad Y_{2}:=X_{2}, \quad Y_{3}:=X_{4}, \quad Y_{4}:=X_{2}+2 X_{3}+2 X_{4}
$$

obtained in Lemma 2.5 form a basis of $\operatorname{Lie}(\mathbb{G})$. Moreover, for all $j=1, \ldots, 4$, we proved that $Y_{j} \mathbb{1}_{E} \geq 0$. These facts will permit us to conclude the proof of Theorem 1.4. Since there is nothing special about the fact that we have a Lie algebra of dimension 4 , we state in full generality the proposition which yields the desired conclusion.

Proposition 2.6. Let $\mathbb{G}$ be any Carnot group. Let $E \subset \mathbb{G}$. Let $Y_{1}, \ldots, Y_{n}$ be a basis of Lie $(\mathbb{G})$. Assume that $Y_{j} \mathbb{1}_{E} \geq 0$, for all $j=1, \ldots, n$. Then $E$ is equivalent to an Euclidean Lipschitz domain.

For the proof of Proposition 2.6, we need to choose a good representative for the set $E$. In fact, we want to have an equivalent set $\tilde{E}$ for which all line flows of $Y_{j}, j=1, \ldots, n$, meet $\tilde{E}$ in a half-line. Such a fact will also be useful for Section 3.1, where we will write $\partial \tilde{E}$ as a graph.

The good representative that we need is the Lebesgue representative of our original set: it allows us to obtain monotonicity along every flow line on every direction $Y_{j}$. Subsequently, we show that the topological boundary of this new set is locally a Lipschitz graph.

Recall that if $X$ is a left-invariant vector field in a Lie group $\mathbb{G}$, i.e., $X \in \operatorname{Lie}(\mathbb{G})$, then its flow is a right translation. Namely,

$$
\Phi_{X}(p, t)=p \exp (t X), \quad \forall p \in \mathbb{G} .
$$

Lemma 2.7. Let $\mathbb{G}$ be any Carnot group. Let $E \subset \mathbb{G}$. Let $X \in \operatorname{Lie}(\mathbb{G})$. Assume that $X \mathbb{1}_{E} \geq 0$. Then, for any $t>0$, we have that (almost everywhere) it holds $\mathbb{1}_{E} \leq \mathbb{1}_{E} \circ \Phi_{X}(\cdot, t)$. In particular, it is true that a.e.

$$
\mathbb{1}_{E \exp (X)} \leq \mathbb{1}_{E}
$$

Proof. Since $X$ is a divergence free vector field on the manifold $\mathbb{G}$, endowed with a Haar measure $\operatorname{vol}_{\mathbb{G}}$, we can prove that: if $u \in L_{\text {loc }}^{1}(\mathbb{G})$ satisfies $X u \geq 0$ in the sense of distributions, then, for all $t>0, u \circ \Phi_{X}(\cdot, t) \geq u \operatorname{vol}_{\mathbb{G}}$-a.e. in $\mathbb{G}$. The statement of the lemma then follows immediately.

What we need to show is that, for any non-negative $g \in C_{c}^{1}(\mathbb{G})$, the map $t \mapsto \int_{\mathbb{G}} g u \circ \Phi_{X}(\cdot, t) d \operatorname{vol}_{\mathbb{G}}$ is increasing in $t$. Indeed, the semigroup property 
of the flow, and the fact that $X$ is divergence-free yield

$$
\begin{aligned}
\int_{\mathbb{G}} g u \circ \Phi_{X}(\cdot, t+s) d \operatorname{vol}_{\mathbb{G}}-\int_{\mathbb{G}} g u \circ \Phi_{X}(\cdot, t) d \operatorname{vol}_{\mathbb{G}} \\
=\int_{\mathbb{G}} u g \circ \Phi_{X}(\cdot,-t-s) d \operatorname{vol}_{\mathbb{G}}-\int_{\mathbb{G}} u g \circ \Phi_{X}(\cdot,-t) d \operatorname{vol}_{\mathbb{G}} \\
=\int_{\mathbb{G}} u g \circ \Phi_{X}\left(\Phi_{X}(\cdot,-s),-t\right) d \operatorname{vol}_{\mathbb{G}}-\int_{\mathbb{G}} u g \circ \Phi_{X}(\cdot,-t) d \operatorname{vol}_{\mathbb{G}} \\
=-s \int_{\mathbb{G}} u X\left(g \circ \Phi_{X}(\cdot,-t)\right) d \operatorname{vol}_{\mathbb{G}}+o(s) \\
=s \int_{\mathbb{G}}\left(g \circ \Phi_{X}(\cdot,-t)\right) X u d \operatorname{vol}_{\mathbb{G}}+o(s),
\end{aligned}
$$

which, recalling that $X u \geq 0$, yields that $t \mapsto \int_{\mathbb{G}} g u \circ \Phi_{X}(\cdot, t) d \operatorname{vol}_{\mathbb{G}}$ is (weakly) increasing in $t$.

Lemma 2.8. Let $\mathbb{G}$ be any Carnot group. Let $E \subset \mathbb{G}$. Let $Y_{1}, \ldots, Y_{k} \in$ $\operatorname{Lie}(\mathbb{G})$. Assume that $Y_{j} \mathbb{1}_{E} \geq 0$, for all $j=1, \ldots, k$. Then there exists $\tilde{E}$ such that $\operatorname{vol}_{\mathbb{G}}(E \Delta \tilde{E})=0$ and, for all $p \in \mathbb{G}$ and $j=1, \ldots, k$, there exists $T \in[-\infty,+\infty]$ such that

$$
\left\{t \in \mathbb{R}: p \exp \left(t Y_{j}\right) \in \tilde{E}\right\}
$$

equals $(T,+\infty)$ or $[T,+\infty)$.

Proof. In a Carnot group, such as $\mathbb{G}$, Haar measures are both left- and right-invariant. In this proof we will make use of the fact that $\operatorname{vol}_{\mathbb{G}}$ is rightinvariant. Flows of left-invariant vector fields are right translations, thus isometries for any right-invariant distance. The balls $B_{r}$ considered in this proof are to be understood with respect to a fixed right-invariant Riemannian metric.

Let $\tilde{E}$ be the Lebesgue representative of $E$, i.e., the set of points having density 1:

$$
x \in \tilde{E} \Leftrightarrow \lim _{r \rightarrow 0} \frac{\operatorname{vol}_{\mathbb{G}}\left(B_{r}(x) \cap E\right)}{\operatorname{vol}_{\mathbb{G}}\left(B_{r}(x)\right)}=1 .
$$

By the Lebesgue-Besicovitch Differentiation Theorem, $\tilde{E}$ and $E$ agree $\operatorname{vol}_{\mathbb{G}}$-a.e. We claim that $\tilde{E}$ fulfils the requirements of Lemma 2.8. Indeed, what we need to prove is: let $p \in \tilde{E}, X$ a left-invariant vector field such that $X \mathbb{1}_{E} \geq 0$ : then for any $t>0$ the point $y=p \exp (t X)$ belongs to $\tilde{E}$.

The vector field $X$ is smooth and the flow $\Phi_{X}(\cdot, t)$ is an isometry for the right-invariant metric, so it sends balls to balls of the same size. In the 
following denote by $y$ the point $\Phi_{X}(\cdot, t)(p)$. We assume $p \in \tilde{E}$, so $\lim _{r \rightarrow 0}$ $\frac{\operatorname{vol}_{\mathbb{G}}\left(B_{r}(p) \cap E\right)}{\operatorname{vol}_{\mathbb{G}}\left(B_{r}(p)\right)}=1$. By the invariance of $\operatorname{vol}_{\mathbb{G}}$ along the flow we have $\operatorname{vol}_{\mathbb{G}}\left(B_{r}(p) \cap E\right)=\operatorname{vol}_{\mathbb{G}}\left(B_{r}(y) \cap E \exp (X)\right)$ and $\operatorname{vol}_{\mathbb{G}}\left(B_{r}(p)\right)=\operatorname{vol}_{\mathbb{G}}\left(B_{r}(y)\right)$. With the aid of Lemma 2.7, we then have $\operatorname{vol}_{\mathbb{G}}\left(B_{r}(p) \cap E\right) \leq \operatorname{vol}_{\mathbb{G}}\left(B_{r}(y) \cap\right.$ $E)$. Altogether we can write

$$
\frac{\operatorname{vol}_{\mathbb{G}}\left(B_{r}(y) \cap E\right)}{\operatorname{vol}_{\mathbb{G}}\left(B_{r}(y)\right)} \geq \frac{\operatorname{vol}_{\mathbb{G}}\left(B_{r}(p) \cap E\right)}{\operatorname{vol}_{\mathbb{G}}\left(B_{r}(p)\right)}
$$

Therefore we have

$$
\lim _{r \rightarrow 0} \frac{\operatorname{vol}_{\mathbb{G}}\left(B_{r}(p) \cap E\right)}{\operatorname{vol}_{\mathbb{G}}\left(B_{r}(p)\right)}=1 \Longrightarrow \lim _{r \rightarrow 0} \frac{\operatorname{vol}_{\mathbb{G}}\left(B_{r}(y) \cap E\right)}{\operatorname{vol}_{\mathbb{G}}\left(B_{r}(y)\right)}=1,
$$

and the lemma is proved.

Remark 2.9. If $Y_{1}, \ldots, Y_{k}$ form a basis of $\operatorname{Lie}(\mathbb{G})$, then the set $\tilde{E}$ is actually open. Indeed, let $q$ be a point on the topological boundary of $E$ and let us show that the upper density of $E$ at $q$ is strictly less than one. Any direction in the convex envelope of some given monotone directions is in turn monotone, thus the whole cone $\hat{Y}$ obtained as convex envelope of $Y_{1}, \ldots, Y_{k}$ is made of monotone directions. Under the assumption that $Y_{1}, \ldots, Y_{k}$ form a basis of Lie $(\mathbb{G})$, this cone has non-empty interior. The complement of $E$ is also a set with constant horizontal normal and contains a sequence of points $q_{n}$ converging to $q$.

By means of Lemma 2.8, the cone $q_{n} \exp (-\hat{Y})$ is all contained in the complement of $E$. Hence, since $q_{n} \rightarrow q$, we have that the interior of $q \exp (-\hat{Y})$ is in the complement of $E$. Since $\hat{Y}$ is an Euclidean cone with non-empty interior, the density of $q \exp (-\hat{Y})$ at $q$ is strictly positive. Therefore, the upper density of $E$ at $q$ must be strictly less than 1 .

Hence, by Lemma 2.5, every set in the Engel group that has normal $X_{2}$ has a representative that is open and satisfies the conclusion of Lemma 2.8.

Proof of Proposition 2.6. By Lemma 2.8 and the remark right after it, we can assume the following: let $E \subset \mathbb{G}$, where $\mathbb{G}$ is a Lie group, let $Y_{1}, \ldots, Y_{n}$ be a basis of $\operatorname{Lie}(\mathbb{G})$ such that for all $p \in \partial E$ and $j=1, \ldots, k$, we have that

$$
\left\{t \in \mathbb{R}: p \exp \left(t Y_{j}\right) \in E\right\}=(0,+\infty)
$$

We want to show that $\partial E$ is locally a Lipschitz graph. 
Fix $p_{0} \in \partial E$. For all $p \in \mathbb{G}$ consider the open set

$$
C_{p}:=\left\{p \exp \left(\sum_{j=1}^{n} \alpha_{j} Y_{j}\right): \alpha_{j}>0\right\} .
$$

Fix a (smooth) coordinate chart $\varphi: U \rightarrow \mathbb{R}^{n}$ from a compact neighbourhood of $p_{0}$. Since $\varphi$ is smooth and $C_{p}$ change smoothly in $p$, then, for all $p \in U$, the set $\varphi\left(C_{p}\right)$ changes smoothly. Thus one can find a fixed Euclidean cone $C \subseteq \mathbb{R}^{n}$ of the form

$$
C:=\left\{\sum_{j=1}^{n} \alpha_{j} v_{j}: \alpha_{j}>0\right\}
$$

for some basis $v_{1}, \ldots, v_{n}$ of $\mathbb{R}^{n}$, such that

$$
\varphi(\partial E) \cap(x+C)=\emptyset, \quad \forall x \in \varphi(\partial E) .
$$

Note that consequently we also have that $\varphi(\partial E) \cap(x-C)=\emptyset$. By a standard argument, e.g., see [1, Theorem 2.61, page 82], one can write $\varphi(\partial E)$ as a graph in any direction $v \in C$ with respect to any hyperplane $\Pi$ such that $\Pi \cap(C \cup-C)=\emptyset$.

Observation 2.10. As a byproduct we get of course that the set $E$ has rectifiable boundary.

\section{Further regularity in a model of the Engel group}

\subsection{Theorem 1.5: entire Lipschitz graphs in coordinates of the second kind}

We devote this section to the proof of Theorem 1.5, which states that a set with constant horizontal normal is, after a suitable choice of exponential coordinates of the second kind, the upper-graph of an entire Lipschitz function. Recall that, by Lemma 2.2, if the normal is $X_{1}$ we have a vertical half-space, hence Theorem 1.5 is immediate in this case. We are thus left with the harder case of normal $X \neq X_{1}$. By Lemma 2.3 we can assume, up to a Lie algebra isomorphism, that the normal is $X_{2}$. After this endomorphism has been performed, we choose exponential coordinates of the second kind as we are about to describe. 
On $\mathbb{R}^{4}$ with coordinates $x_{1}, x_{2}, x_{3}, x_{4}$, we consider the following vector fields:

$$
\begin{aligned}
& X_{1}=\partial_{1}, \\
& X_{2}=\partial_{2}+x_{1} \partial_{3}+\frac{x_{1}^{2}}{2} \partial_{4}, \\
& X_{3}=\partial_{3}+x_{1} \partial_{4}, \\
& X_{4}=\partial_{4} .
\end{aligned}
$$

Such vector fields form a Lie algebra which is 4-dimensional. Their only non-trivial brackets are

$$
\left[X_{1}, X_{2}\right]=X_{3}, \quad\left[X_{1}, X_{3}\right]=X_{4}
$$

Therefore, such an algebra is isomorphic to the Engel Lie algebra. Using the general theory of (nilpotent) Lie groups one can prove that there exists a (unique) product on $\mathbb{R}^{4}$ for which the vector fields $X_{1}, X_{2}, X_{3}, X_{4}$ are leftinvariant (and therefore a basis of the Lie algebra).

The coordinates for the Engel group that we are using are called the exponential coordinates of the second kind. Namely, if $X_{1}, X_{2}, X_{3}, X_{4}$ are a basis of the Lie algebra that satisfies (3.2), then the map

$$
\left(x_{1}, x_{2}, x_{3}, x_{4}\right) \mapsto \exp \left(x_{4} X_{4}\right) \exp \left(x_{3} X_{3}\right) \exp \left(x_{2} X_{2}\right) \exp \left(x_{1} X_{1}\right)
$$

is a diffeomorphism between $\mathbb{R}^{4}$ and the Engel group. Moreover, the vectors $X_{1}, X_{2}, X_{3}, X_{4}$ are pulled back to $\mathbb{R}^{4}$ to the vector fields as defined in (3.1).

Recall that in a Lie group $G$ there is a differential geometric interpretation for the product between an element $p \in G$ with the image $\exp (t X)$ of a multiple of a left-invariant vector field $X$ via the exponential map. Indeed, one has the formula

$$
p \cdot \exp (t X)=\Phi_{X}^{t}(p)
$$

where $\Phi_{X}^{t}(p)$ denotes the flow of $X$ after time $t$ starting from $p$.

With the discussion at the beginning of this subsection in mind, the aim is now to study those sets (in the model (3.1)) that are invariant in the direction of $X_{1}$ and are monotone in the direction of $X_{2}$. Namely, let $E \subseteq \mathbb{R}^{4}$ be an open set (we always take the Lebesgue representative), we say that $E$ is $X_{2}$-calibrated if the following two properties holds:

$X_{1}$-invariance: if $p \in E$ then, for any $t \in \mathbb{R}, p \exp \left(t X_{1}\right) \in E$; 
$X_{2}$-monotonicity: for all $p \in \mathbb{R}^{4}$, the set $\left\{t \in \mathbb{R}: p \exp \left(t X_{2}\right) \in E\right\}$ is an open half-line of the form $(T,+\infty)$ for some $T \in\{-\infty\} \cup \mathbb{R} \cup\{+\infty\}$.

Therefore, if $E$ is an $X_{2}$-calibrated set then $E$ has constant normal equal to $X_{2}$, i.e., $X_{2} \mathbb{1}_{E} \geq 0$ and $X_{1} \mathbb{1}_{E}=0$. Vice versa, by Lemma 2.8 and Remark 2.9, any set $E$ with normal $X_{2}$ admits an $X_{2}$-calibrated set $\tilde{E}$ that is equivalent to $E$.

By formula (3.3), we can calculate a product $p \cdot \exp (t X)$ without knowing an explicit formula for the product. Let us consider the two cases when $X$ is $X_{1}$ or $X_{2}$ as above.

Regarding the flow of $X_{1}$, we need to solve the ODE

$$
\left\{\begin{array}{l}
\gamma(0)=p, \\
\dot{\gamma}(t)=\left(X_{1}\right)_{\gamma(t)} .
\end{array}\right.
$$

Writing $\gamma=\left(\gamma_{1}, \gamma_{2}, \gamma_{3}, \gamma_{4}\right)$ and using the definition of $X_{1}$, the second inequality becomes $\left(\dot{\gamma}_{1}(t), \dot{\gamma}_{2}(t), \dot{\gamma}_{3}(t), \dot{\gamma}_{4}(t)\right)=\partial_{1}=(1,0,0,0)$. Integrating, we have

$$
\gamma_{1}(t)=p_{1}+t, \quad \gamma_{2}(t)=p_{2}, \quad \gamma_{3}(t)=p_{3}, \quad \gamma_{4}(t)=p_{4} .
$$

Thus,

$$
p \cdot \exp \left(t X_{1}\right)=\left(p_{1}+t, p_{2}, p_{3}, p_{4}\right) .
$$

Regarding the flow of $X_{2}$, we consider the ODE

$$
\left\{\begin{array}{l}
\gamma(0)=p \\
\dot{\gamma}(t)=\left(X_{2}\right)_{\gamma(t)}=\left(0,1, \gamma_{1}(t),\left(\gamma_{1}(t)\right)^{2} / 2\right) .
\end{array}\right.
$$

Integrating, we have

$$
\gamma_{1}(t)=p_{1}, \quad \gamma_{2}(t)=p_{2}+t, \quad \gamma_{3}(t)=p_{3}+p_{1} t, \quad \gamma_{4}(t)=p_{4}+p_{1}^{2} t / 2 .
$$

Thus,

$$
p \cdot \exp \left(t X_{2}\right)=\left(p_{1}, p_{2}+t, p_{3}+p_{1} t, p_{4}+p_{1}^{2} t / 2\right) .
$$

Thus, we replace the previous definition:

Definition 3.7 ( $X_{2}$-calibration). An open set $E \subseteq \mathbb{R}^{4}$ is called $X_{2^{-}}$ calibrated if

(i): if $p \in E$ then, for any $t \in \mathbb{R}, p+(t, 0,0,0) \in E$; 
(ii): for all $p \in \mathbb{R}^{4}$ there exists $T \in\{-\infty\} \cup \mathbb{R} \cup\{+\infty\}$ such that

$$
\left\{t \in \mathbb{R}: p+\left(0, t, p_{1} t, p_{1}^{2} t / 2\right) \in E\right\}=(T,+\infty)
$$

Since the set $E$ is assumed to be open, condition ii) is equivalent to the following condition:

(ii'):

$$
p \in E, t>0 \Longrightarrow p_{t}:=p+\left(0, t, p_{1} t, p_{1}^{2} t / 2\right) \in E
$$

Example 3.8. Let $g: \mathbb{R} \rightarrow \mathbb{R}$ be a non-increasing and upper semicontinuous function. Consider the set

$$
E:=\left\{x \in \mathbb{R}^{4}: x_{2}>g\left(x_{4}\right)\right\}
$$

Since $g$ is assumed upper semicontinuous, then $E$ is an open set. Then we claim that the set $E$ is $X_{2}$-calibrated. Indeed, such a fact can be seen as a consequence of Theorem 3.17 from next section in which we give a characterization of sets with constant normal. However, we present here a direct and detailed proof of such a claim. Property (i) is obvious, for such an $E$, since in the definition of $E$ the variable $x_{1}$ does not appear.

Let us show property (ii'). If $p \in E$, then $p_{2}>g\left(p_{4}\right)$. Now, if $t>0$, we have that $p_{2}+t>p_{2}$ and $g\left(p_{4}+p_{1}^{2} t\right) \leq g\left(p_{4}\right)$, being $g$ non-increasing. Thus, $p_{2}+t-g\left(p_{4}+p_{1}^{2} t\right) \geq p_{2}-g\left(p_{4}\right)>0$ and so $p+\left(0, t, p_{1} t, p_{1}^{2} t\right) \in E$. QED

Now we provide some intermediate steps that will be needed to complete the proof of Theorem 1.5.

Lemma 3.9. Consider $\mathbb{R}^{4}$ endowed with the vector fields (3.1). Let $E \subset \mathbb{R}^{4}$ be an open $X_{2}$-calibrated set. Denote by $\overline{\mathbb{R}}$ the extended real line, i.e., $\overline{\mathbb{R}}:=$ $\{-\infty\} \cup \mathbb{R} \cup\{+\infty\}$. Then there exists an upper semicontinuous function $G$ : $\mathbb{R}^{2} \rightarrow \overline{\mathbb{R}}$ such that

$$
E=\left\{x \in \mathbb{R}^{4}: x_{2}>G\left(x_{3}, x_{4}\right)\right\}
$$

Proof. For each $x_{3}, x_{4} \in \mathbb{R}$, define $G\left(x_{3}, x_{4}\right):=\inf \left\{x_{2}:\left(0, x_{2}, x_{3}, x_{4}\right) \in E\right\}$. Here $\inf \{\emptyset\}=+\infty$. Whenever such an infimum is finite, then it is not 
realized, since $E$ is open. Since $E$ is $X_{2}$-monotone and $\left(0, x_{2}, x_{3}, x_{4}\right)$. $\exp \left(t X_{2}\right)=\left(0, x_{2}+t, x_{3}, x_{4}\right)$, we have that

$$
E \cap\left(\{0\} \times \mathbb{R} \times\left\{x_{3}\right\} \times\left\{x_{4}\right\}\right)=\{0\} \times\left(G\left(x_{3}, x_{4}\right),+\infty\right) \times\left\{x_{3}\right\} \times\left\{x_{4}\right\} .
$$

For any $x \in \mathbb{R}^{4}$, since $E$ is $X_{1}$-invariant, we have that

$$
x \in E \Longleftrightarrow\left(0, x_{2}, x_{3}, x_{4}\right) \in E \Longleftrightarrow x_{2}>G\left(x_{3}, x_{4}\right) .
$$

The upper semicontinuity of $G$ follows because $E$ is open.

Lemma 3.10. Let $\mathbb{G}$ be the Engel group in exponential coordinates of second kind with Lie algebra as in (3.1). Let $E \subset \mathbb{G}$ be an open $X_{2}$-calibrated set. Assume that there exists $\tilde{p} \in E$ such that $\tilde{p}+(0, \mathbb{R}, 0,0) \in E$. Then

$$
\left\{x \in \mathbb{R}^{4}: x_{3} \neq \tilde{p}_{3}, x_{4}>\tilde{p}_{4}\right\} \subseteq E .
$$

Proof. Let $x \in \mathbb{R}^{4}$ with $x_{3} \neq \tilde{p}_{3}$ and $x_{4}>\tilde{p}_{4}$. Set $s:=x_{3}-\tilde{p}_{3}$, which is nonzero, and $t:=\frac{s^{2}}{x_{4}-\tilde{p}_{4}}$, which is positive. By the particular assumption on $\tilde{p}$, we have

$$
\left(\tilde{p}_{1}, x_{2}-t, \tilde{p}_{3}, \tilde{p}_{4}\right) \in E .
$$

By $X_{1}$-invariance,

$$
\left(s / t, x_{2}-t, \tilde{p}_{3}, \tilde{p}_{4}\right) \in E .
$$

By $X_{2}$-monotonicity,

$$
\left(\frac{s}{t}, x_{2}-t+t, \tilde{p}_{3}+\frac{s}{t} t, \tilde{p}_{4}+\frac{s^{2}}{t^{2}} t\right) \in E .
$$

Explicitly,

$$
\begin{aligned}
\left(\frac{s}{t}, x_{2}, \tilde{p}_{3}+s, \tilde{p}_{4}+\frac{s^{2}}{t}\right) & =\left(\frac{s}{t}, x_{2}, \tilde{p}_{3}+x_{3}-\tilde{p}_{3}, \tilde{p}_{4}+s^{2} \frac{x_{4}-\tilde{p}_{4}}{s^{2}}\right) \\
& =\left(\frac{s}{t}, x_{2}, x_{3}, x_{4}\right) \in E .
\end{aligned}
$$

By $X_{1}$-invariance,

$$
\left(x_{1}, x_{2}, x_{3}, x_{4}\right) \in E .
$$


Observation 3.11. The previous lemma is saying that the function $G$ describing $E$ has the property that the closure of the level set $G^{-1}(-\infty)$ is a half-space orthogonal to $x_{4}$. With an analogous argument we can actually prove the stronger statement: if $\left(p_{1}^{n}, p_{3}^{n}, p_{4}^{n}\right) \rightarrow\left(p_{1}, p_{3}, p_{4}\right)$ as $n \rightarrow \infty$ and $G\left(p_{1}^{n}, p_{3}^{n}, p_{4}^{n}\right) \rightarrow-\infty$ then on the half-space $\left\{x_{4}>p_{4}\right\}$ the function $G$ must take the value $-\infty$. We skip the proof of this statement, since it will easily follow from the properties of the set $C$ described in Example 3.31.

Definition 3.12 (Partially Lipschitz map). Let $G: \mathbb{R}^{k} \rightarrow \overline{\mathbb{R}}, v \in \mathbb{R}^{k}$, and $L>0$. We say that $G$ is partially L-Lipschitz along $v$ if, for all $t>0$ and $x \in \mathbb{R}^{k}$, one has

$$
G(x+t v) \leq L t+G(x)
$$

Note that in the above definition we only have a condition for positive $t$ and also for the difference $G(x+t v)-G(x)$, not for the absolute value. Example of partially Lipschitz maps are the monotone maps. Indeed, every non-increasing function $G: \mathbb{R} \rightarrow \mathbb{R}$ is partially $L$-Lipschitz along $v$, for all $L>0$ and all $v>0$.

Lemma 3.13. Let $G: \mathbb{R}^{2} \rightarrow \overline{\mathbb{R}}$ be such that the set $E=\left\{x \in \mathbb{R}^{4}: x_{2}>\right.$ $\left.G\left(x_{3}, x_{4}\right)\right\}$ is $X_{2}$-monotone. Then $G$ is partially 1 -Lipschitz along any vector $\left(a, a^{2} / 2\right)$, with $a \in \mathbb{R}$.

Proof. Fix $x_{3}, x_{4} \in \mathbb{R}$. Assume $G\left(x_{3}, x_{4}\right) \neq+\infty$, otherwise there is nothing to prove. Take $x_{2}>G\left(x_{3}, x_{4}\right)$. Thus $\left(a, x_{2}, x_{3}, x_{4}\right) \in E$. Since $E$ is $X_{2}$-monotone, we have that, for all $t>0$,

$$
\left(a, x_{2}+t, x_{3}+a t, x_{4}+\frac{a^{2}}{2} t\right) \in E .
$$

So $x_{2}+t>G\left(x_{3}+a t, x_{4}+a^{2} t / 2\right)$, for all $t>0$. Letting $x_{2} \rightarrow G\left(x_{3}, x_{4}\right)$, we get

$$
G\left(x_{3}, x_{4}\right)+t \geq G\left(x_{3}+a t, x_{4}+a^{2} t / 2\right),
$$

which ends the proof.

As a consequence we get the following corollary, which concludes the proof of Theorem 1.5. Indeed, recall that (as discussed in the beginning of this subsection) the case of normal $X=X_{1}$ is easily handled, while for the 
case of normal $X \neq X_{1}$ we have chosen coordinates of the second kind (3.1) in such a way that the normal is $X_{2}$.

Corollary 3.14. There exist coordinates in which the set $E$ of constant normal $\mathrm{X}_{2}$ can be expressed as upper-graph of a globally Lipschitz function of $\mathbb{R}^{3}$.

Proof. From the previous lemma, for any direction $v=\left(v_{1}, v_{2}\right)$ in $\mathbb{R}_{x_{3}, x_{4}}^{2}$ with $|v|=1$ and $v_{2}>0$, the function $G$ is $\frac{\left(v_{1}\right)^{2}}{2 v_{2}}$ partially Lipschitz along $v$. So at every point $y$ on the graph of $G$ there is a cone-shaped domain $y+\left\{\left(x_{1}, x_{2}, x_{3}, x_{4}\right): x_{4} \geq 0, x_{2}>\frac{\left(x_{3}\right)^{2}}{2 x_{4}} \sqrt{x_{3}^{2}+x_{4}^{2}}\right\}$ that is contained in the upper-graph of $G$.

Remark that the cone is independent of the point $y$ on the graph of $G$, it is just moved via (Euclidean) translations. By suitably rotating coordinates, we can make therefore $E$ to be the upper-graph of a globally Lipschitz function: namely we have to choose a graphing direction that lies in the interior of the set $\left\{\left(x_{1}, x_{2}, x_{3}, x_{4}\right): x_{4} \geq 0, x_{2}>\frac{\left(x_{3}\right)^{2}}{2 x_{4}} \sqrt{x_{3}^{2}+x_{4}^{2}}\right\}$.

\subsection{Analytic characterization and examples of sets with normal $\boldsymbol{X}_{2}$}

We present in this subsection a characterization (as well as some examples) of sets with normal $X_{2}$ in the model (3.1) of the Engel group that we have used above. Recall again that there is no loss of generality in restricting to this particular case, since any set with constant horizontal normal $X \neq X_{1}$ can be brought to this case (while for sets with normal $X_{1}$ we have a much easier characterization, since they are half-spaces by Lemma 2.2).

We recall a few facts on BV functions, with reference to [18, pages 354$379]$.

Let $u$ be an $L_{\text {loc }}^{1}$ function on $\mathbb{R}^{n}$; the subgraph $S U$ of $u$, i.e., the set $\left\{(x, y) \in \mathbb{R}^{n} \times \mathbb{R}: y<u(x)\right\}$, is a set of locally finite (Euclidean) perimeter if and only if $u$ is $B V_{\text {loc }}$ (Thm. 1 page 371 ).

Let $u: \mathbb{R}^{n} \rightarrow \mathbb{R}$ be $L_{\text {loc }}^{1}$. Define the approximate limsup and liminf at $x \in \mathbb{R}^{n}$ respectively as follows, where for any $t \in \mathbb{R}$ we use the notation $U_{t, u}:=\left\{x \in \mathbb{R}^{n}: u(x)>t\right\}$ and $L_{t, u}:=\left\{x \in \mathbb{R}^{n}: u(x)<t\right\}:$

$u_{+}(x):=\sup \left\{t \in \mathbb{R}:\right.$ the $n$-dim. density of the set $L_{t, u}$ at $x$ is 0$\}$, $u_{-}(x):=\inf \left\{t \in \mathbb{R}:\right.$ the $n$-dim. density of the set $U_{t, u}$ at $x$ is 0$\}$. 
When $u_{+}(x)=u_{-}(x)$ we say that $x$ is a point of "approximate continuity" for $u$. The set $J$ of points where the strict inequality $u_{+}(x)>u_{-}(x)$ holds is the "jump set" of $u$. Then we have (see [18, pages 355]): the set $J$ is $\mathcal{H}^{n-1}$-measurable and countably $\mathcal{H}^{n-1}$-rectifiable.

The term "jump set" is justified by the result we are about to recall. Denote, for $x \in \mathbb{R}^{n}$ and $\nu_{x} \in S^{n-1}$, the half-space $\left\{y \in \mathbb{R}^{n}:\langle y-x, \nu\rangle>0\right\}$ by $E^{+}(x, \nu)$. Analogously denote the half space $\left\{y \in \mathbb{R}^{n}:\langle y-x, \nu\rangle<0\right\}$ by $E^{-}(x, \nu)$.

For $\mathcal{H}^{n-1}$-a.e. $x$ in $J$ there exists a (unique) $\nu_{x} \in S^{n-1}$ such that it holds:

$$
\operatorname{aplim}_{y \rightarrow x, y \in E^{+}(x, \nu)} u(y)=u_{+}(x) \text { and } \operatorname{aplim}_{y \rightarrow x, y \in E^{-}(x, \nu)} u(y)=u_{-}(x) .
$$

The notion of approximate limit here (see [18, pages 210]) is meant as follows:

for all $\epsilon>0$ the set $\left\{y \in E^{+}(x, \nu):\left|u(y)-u_{+}(x)\right| \geq \epsilon\right\}$ has $n$-dim. density 0 at the point $x$. Analogously for all $\epsilon>0$ the set $\left\{y \in E^{-}(x, \nu)\right.$ : $\left.\left|u(y)-u_{-}(x)\right| \geq \epsilon\right\}$ has $n$-dim. density 0 at the point $x$.

Then we can improve our knowledge of $J$ with the following statement ([18, pages 355]): the set $J$ is $\mathcal{H}^{n-1}$-measurable and countably $\mathcal{H}^{n-1}$ rectifiable; moreover on $J$ we have that the approximate tangent space (in the sense of geometric measure theory) exists for $\mathcal{H}^{n-1}$-a.e. $x$ and is given by the orthogonal to $\nu_{x}$.

Regarding the distributional derivative $D u$ of the $B V_{\text {loc }}$ function $u$ : $\mathbb{R}^{n} \rightarrow \mathbb{R}$, we know that it is a locally finite vector-valued measure (by definition). Its components will be denoted by $\partial_{1} u, \ldots, \partial_{n} u$. Setting $D^{(j)} u:=$ $D u\left\llcorner J\right.$ and $\tilde{D} u:=D u-D^{(j)} u$ we are going to use the splitting $D u=\tilde{D} u+$ $D^{(j)} u$. The measures $D^{(j)} u$ and $\tilde{D} u$ are mutually singular. For the partial derivatives, i.e., the components of the vector $D u$, we will make use of the corresponding notations $D^{(j)} u=\left(\partial_{1}^{(j)} u, \ldots, \partial_{n}^{(j)} u\right)$ and $\tilde{D} u=$ $\left(\tilde{\partial}_{1} u, \ldots, \tilde{\partial}_{n} u\right)$. There exists (see $\left.[1,18]\right)$ a further splitting of $\tilde{D} u$ into an absolutely continuous (w.r.t. Lebesgue measure) part and a "Cantor part," but we are not going to need it for our purposes. The measure $D^{(j)} u$ is just $\left(u_{+}(x)-u_{-}(x)\right)\left(\mathcal{H}^{1}\llcorner J) \otimes \nu_{x}\right.$.

By recalling Theorems 2 and 3 on page 375 of [18] we will now see how to express the distributional derivative $D \mathbb{1}_{S U}$ of the characteristic function $\mathbb{1}_{S U}$, for $u \in B V_{\text {loc }}$, in terms of $D u$.

We split

$$
D \mathbb{1}_{S U}=D^{(j)} \mathbb{1}_{S U}+D^{(\mathrm{cont})} \mathbb{1}_{S U},
$$

where $D^{(j)} \mathbb{1}_{S U}:=D \mathbb{1}_{S U}\left\llcorner(J \times \mathbb{R})\right.$ and $D^{(\mathrm{cont})} \mathbb{1}_{S U}:=D \mathbb{1}_{S U}-D^{(j)} \mathbb{1}_{S U}$. 
Let $(x, y)$ denote the coordinates for $\mathbb{R}^{n} \times \mathbb{R}$. Then it holds, for $D^{\text {(cont) }} \mathbb{1}_{S U}$ :

$$
\begin{gathered}
\left(D_{i}^{\text {(cont) }} \mathbb{1}_{S U}\right)(\phi(x, y))=\int_{\mathbb{R}^{n} \backslash J} \phi\left(x, u_{+}(x)\right) D_{i} u \text { for any } \phi \in C_{c}^{\infty}\left(\mathbb{R}^{n} \times \mathbb{R}\right) \\
\quad \text { and } i \in\{1,2, \ldots n\}
\end{gathered}
$$

$$
\left(D_{n+1}^{(\mathrm{cont})} \mathbb{1}_{S U}\right)(\phi(x, y))=-\int_{\mathbb{R}^{n}} \phi\left(x, u_{+}(x)\right) d x \text { for any } \phi \in C_{c}^{\infty}\left(\mathbb{R}^{n} \times \mathbb{R}\right) .
$$

Regarding the jump part we have that in $\mathbb{R}^{n} \times \mathbb{R}$

$$
D^{(j)} \mathbb{1}_{S U}=\left(\mathcal{H}^{n}\llcorner V) \otimes\left(\nu_{x}, 0\right)\right.
$$

where $V=\left\{(x, y) \in \mathbb{R}^{n} \times \mathbb{R}: x \in J, u_{-}(x)<y<u_{+}(x)\right\}$ and the vector $\nu_{x}$ is the normal to $J$ in $\mathbb{R}^{n}$.

We are now ready to state the analytic characterization of constant normal sets in the Engel group:

Theorem 3.17. In our model of the Engel group (3.1) a set has finite perimeter and constant horizontal normal $X_{2}$ if and only if it is of the form $\left\{x_{2}>G\left(x_{3}, x_{4}\right)\right\}$ for an upper semicontinuous function $G: \mathbb{R}^{2} \rightarrow \overline{\mathbb{R}}$ with the following properties:

(i): the closure of $\left\{\left(x_{3}, x_{4}\right): G\left(x_{3}, x_{4}\right)=-\infty\right\}$ is a half-space of the form $\left\{\left(x_{3}, x_{4}\right): x_{4} \geq b\right\}$ for some $b \in \overline{\mathbb{R}}$;

(ii): the restriction of $G$ to the open set

$\mathcal{G}:=\mathbb{R}^{2} \backslash \overline{\left\{\left(x_{3}, x_{4}\right): x_{4} \geq b\right\}} \backslash\left\{\left(x_{3}, x_{4}\right): G\left(x_{3}, x_{4}\right)=+\infty\right\}$ is $B V_{\text {loc }}(\mathcal{G})$;

(iii): $G$ satisfies the following partial differential inequality on $\mathcal{G}$ : for all $h \in C_{c}^{\infty}(\mathcal{G})$ such that $h \geq 0$, it holds

$$
\left(\left\langle\partial_{3} G, h\right\rangle\right)^{2}+2\left\langle\partial_{4} G, h\right\rangle\left\langle\mathcal{L}^{2}, h\right\rangle \leq 0
$$

Here $\mathcal{L}^{2}$ denotes the Lebesgue measure on $\mathbb{R}^{2}$ and $\langle$,$\rangle denotes the pair-$ ing of distributions and smooth test functions.

Observation 3.19. The inequality (3.18) can be equivalently expressed by requiring that, for any $h \in C_{c}^{\infty}(\mathcal{G})$ such that $h \geq 0$ and $\int h=1$, it holds

$$
\left(\int_{\mathcal{G}} G \frac{\partial h}{\partial x_{3}}\right)^{2} \leq 2 \int_{\mathcal{G}} G \frac{\partial h}{\partial x_{4}}
$$


Observation 3.21. The partial differential inequality (3.18) is the distributional analogue of the inequality $\left(\partial_{3} G\right)^{2}+2 \partial_{4} G \leq 0$ in the case that $G$ is a smooth function. Indeed, assuming $\left(\partial_{3} G\right)^{2}+2 \partial_{4} G \leq 0$, for any $h \in C_{c}^{\infty}(\mathcal{G})$ such that $h \geq 0$ and $\int h=1$, we have

$$
\int\left(\partial_{3} G\right)^{2} h+2 \int \partial_{4} G h \leq 0 .
$$

Jensen's inequality applied with respect to the measure of unit mass $h d \mathcal{L}^{2}$ yields

$$
\left(\int \partial_{3} G h\right)^{2} \leq \int\left(\partial_{3} G\right)^{2} h
$$

On the other hand, by assuming (3.18) and using it on a sequence of test functions $h_{n}$ having unit integral and converging to the Dirac delta at a point, we pointwise obtain the inequality $\left(\partial_{3} G\right)^{2}+2 \partial_{4} G \leq 0$.

Before proving Theorem 3.17, we first show the following result.

Lemma 3.22. Let $G: \mathcal{G} \subset \mathbb{R}^{2} \rightarrow \mathbb{R}$ be as in Theorem 3.17 and be $J \subset \mathbb{R}^{2}$ its jump part. We take $G$ to be a function of the variables $x_{3}$ and $x_{4}$ and we will denote by $\partial_{3}$ (resp. $\partial_{4}$ ) the partial derivative, which is a Radon measure, with respect to the variable $x_{3}$ (resp. $x_{4}$ ).

The partial differential inequality (3.18) splits into (and actually is equivalent to)

$$
\left(\left\langle\tilde{\partial}_{3} G, h\right\rangle\right)^{2}+2\left\langle\tilde{\partial}_{4} G, h\right\rangle\left\langle\mathcal{L}^{2}, h\right\rangle \leq 0, \quad \partial_{3}^{(j)} G=0,
$$

where we are using the notation $\tilde{\partial}$ and $\partial^{(j)}$ introduced before and $h$ is any non-negative test function. The second equation in (3.23) is equivalent to saying that $J$ has normal $\nu_{x}$ that is parallel to the $x_{4}$-direction for $\mathcal{H}^{1}$-a.e. $x \in J$.

Proof of Lemma 3.22. We shall prove that (3.18) yields the two inequalities in $(3.23)$.

By definition of $\mathcal{H}^{n-1}$-rectifiable we have $J=\cup_{i=1}^{\infty} f_{i}\left(K_{i}\right)$, where the $K_{i}$ 's are compact sets in $\mathbb{R}$ and $f_{i}$ 's are Lipschitz functions from $\mathbb{R}$ to $\mathcal{G}$. We can assume the union $\cup_{i=1}^{\infty} f_{i}\left(K_{i}\right)$ to be disjoint. Fix any $\epsilon>0$ : for each $N$ we can find an open neighbourhood $A_{N, \epsilon}$ of the compact set $\cup_{i=1}^{N} f_{i}\left(K_{i}\right)$ such 
that $\mathcal{L}^{2}\left(A_{N, \epsilon}\right) \leq \epsilon$. This is achieved by taking neighbourhoods of each $f_{i}\left(K_{i}\right)$ having measure at most $\frac{\epsilon}{2^{i}}$ and taking their union from $i=1$ to $i=N$. The fact that we can find an open neighbourhood of $f_{i}\left(K_{i}\right)$ having arbitrarily small area is a consequence of the fact that $f_{i}\left(K_{i}\right)$ has finite $\mathcal{H}^{1}$-measure.

Choose now, for $N$ and $\epsilon$ fixed, a smooth bump function $\psi_{N, \epsilon}$ that is identically 1 on the compact set $\cup_{i=1}^{\infty} f_{i}\left(K_{i}\right)$, identically 0 outside of $A_{N, \epsilon}$ and takes values between 0 and 1 .

Choose any $h \in C_{c}^{\infty}(\mathcal{G})$. The partial differential inequality (3.18) used on the function $h_{N, \epsilon}:=h \psi_{N, \epsilon}$ reads

$$
\begin{aligned}
& \left(\left\langle\tilde{\partial}_{3} G, h_{N, \epsilon}\right\rangle+\left\langle\partial_{3}^{(j)} G, h_{N, \epsilon}\right\rangle\right)^{2}+2\left\langle\tilde{\partial}_{4} G, h_{N, \epsilon}\right\rangle\left\langle\mathcal{L}^{2}, h_{N, \epsilon}\right\rangle \\
& \quad+2\left\langle\partial_{4}^{(j)} G, h_{N, \epsilon}\right\rangle\left\langle\mathcal{L}^{2}, h_{N, \epsilon}\right\rangle \leq 0 .
\end{aligned}
$$

Keeping $N$ fixed and letting $\epsilon \rightarrow 0$, we get that

$$
\tilde{\partial}_{3} G\left(A_{N, \epsilon}\right) \rightarrow \tilde{\partial}_{3} G\left(\cup_{i=1}^{N} f_{i}\left(K_{i}\right)\right)=0,
$$

where the convergence holds since $\cup_{i=1}^{N} f_{i}\left(K_{i}\right)=\cap_{\epsilon>0} A_{N, \epsilon}, \tilde{\partial}_{3} G$ is a Radon measure, and the sets $A_{N, \epsilon}$ are bounded. This, together with an analogous convergence for $\tilde{\partial}_{4} G$ and $\mathcal{L}^{2}$, gives that for $N$ fixed and $\epsilon \rightarrow 0$ :

$$
\left\langle\tilde{\partial}_{3} G, h_{N, \epsilon}\right\rangle \rightarrow 0, \quad\left\langle\tilde{\partial}_{4} G, h_{N, \epsilon}\right\rangle \rightarrow 0, \quad\left\langle\mathcal{L}^{2}, h_{N, \epsilon}\right\rangle \rightarrow 0 .
$$

Let us now look at the remaining terms in (3.24), namely those involving the "jump parts". Denote by $\nu_{3, N}$ the measure $\partial_{3}^{(j)} G\left\llcorner\left(\cup_{i=1}^{N} f_{i}\left(K_{i}\right)\right)\right.$. In the same fashion let $\nu_{4, N}:=\partial_{4}^{(j)} u\left\llcorner\left(\cup_{i=1}^{N} f_{i}\left(K_{i}\right)\right)\right.$. Remark that $\nu_{3, N} \rightarrow \partial_{3}^{(j)} G$ and $\nu_{4, N} \rightarrow \partial_{4}^{(j)} G$, as $N \rightarrow \infty$.

Moreover, for a fixed $N$, we get (recall that $\psi_{N, \epsilon}=1$ on $\cup_{i=1}^{N} f_{i}\left(K_{i}\right)$ ) that, as $\epsilon \rightarrow 0$ :

$$
\left\langle\partial_{3}^{(j)} G, h_{N, \epsilon}\right\rangle \rightarrow\left\langle\nu_{3, N}, h\right\rangle, \quad\left\langle\partial_{4}^{(j)} G, h_{N, \epsilon}\right\rangle \rightarrow\left\langle\nu_{4, N}, h\right\rangle .
$$

So we can send (3.24) to the limit for $\epsilon \rightarrow 0$ and get

$$
\left(\left\langle\nu_{3, N}, h\right\rangle\right)^{2} \leq 0
$$

which holds for every $h \geq 0$. Using the convergence of measures $\nu_{3, N} \rightarrow$ $\partial_{3}^{(j)} G$, as $N \rightarrow \infty$, we obtain that $\partial_{3}^{(j)} G=0$, as in (3.23). This equivalently means that $J$ has a normal $\nu$ always parallel to the $x_{4}$ direction. 
In order to get the first inequality in (3.23) we can use an analogous argument, this time using $1-\psi_{N, \epsilon}$ instead of $\psi_{N, \epsilon}$.

Observation 3.26. The condition on the shape of $J$ is actually equivalent to

$$
\mathcal{H}^{1}\left(J \backslash \cup_{i=1}^{\infty} B_{i}\right)=0
$$

where each $B_{i}$ is a Borel subset of a line parallel to $x_{3}$.

Observation 3.27. It is easily seen that, for $G \in B V_{\mathrm{loc}}$, equations (3.23) are actually equivalent to (3.18).

Proof of Theorem 3.17. As we saw in Lemma 3.9, every set having locally finite horizontal perimeter and constant horizontal normal equal to $X_{2}$ is the upper-graph of a function $G: \mathbb{R}^{2} \rightarrow \overline{\mathbb{R}}$ of the variables $\left(x_{3}, x_{4}\right)$. Such $G$ is upper semicontinuous and by Lemma 3.10 the closure of the level set at $-\infty$ is a (closed) half-space in the direction $x_{4}$. Such a $G$ will then be $L_{\text {loc }}^{1}$ on the open set $\mathcal{G}$ (see Observation 3.11).

We have moreover seen that $E$ has Lipschitz boundary (in the Euclidean sense) when we choose suitable coordinates (Lemma 3.13). This makes it a set of locally finite Euclidean perimeter. Thus, since being of locally finite Euclidean perimeter is a notion that is independent of coordinates, going back to the original coordinates the function $G$ must be $B V_{\text {loc }}$ on $\mathcal{G}$.

We thus need to prove that, for $G$ as in assumptions (i) and (ii), the set $E:=\left\{x \in \mathbb{R}^{4}: x_{2}>G\left(x_{3}, x_{4}\right)\right\}$ is $X_{2}$-monotone if and only if $G$ satisfies (3.18).

Regarding $X_{2}$-monotonicity, we split the derivatives $\partial_{x_{j}} \mathbb{1}_{E}$ in the "approximately continuous part" and the "jump part".

We can compute, on the "approximately continuous part" $\left(\mathbb{R}_{x_{1}, x_{3}, x_{4}}^{3}\right)$ $\left.\left(\mathbb{R}_{x_{1}} \times J\right)\right) \times \mathbb{R}_{x_{2}}$, the horizontal normal to $\mathbb{1}_{E}$ as follows: for any nonnegative $h \in C_{c}^{\infty}\left(\mathbb{R}^{4}\right)$ it holds (from (3.15))

$$
\begin{aligned}
& {\left[\left(\partial_{2}+x_{1} \partial_{3}+\frac{x_{1}^{2}}{2} \partial_{4}\right)^{\mathrm{cont}} \mathbb{1}_{E}\right](h)} \\
& =\int_{\mathbb{R}_{x_{1}, x_{3}, x_{4}}^{3} \backslash\left(\mathbb{R}_{x_{1}} \times J\right)} h\left(x_{1}, G\left(x_{3}, x_{4}\right), x_{3}, x_{4}\right) \\
& \quad \times\left[1-x_{1}\left(\partial_{3} G\right)\left(x_{3}, x_{4}\right)-\frac{x_{1}^{2}}{2}\left(\partial_{4} G\right)\left(x_{3}, x_{4}\right)\right] .
\end{aligned}
$$


We now consider $J \times \mathbb{R}$ and (recall (3.16)) here we have $\partial_{2} \mathbb{1}_{E}=0$. Let further $(a, b)$ be the vector $\left(\partial_{3} \mathbb{1}_{E}, \partial_{4} \mathbb{1}_{E}\right)$. Then

$$
\left(\partial_{2}+x_{1} \partial_{3}+\frac{x_{1}^{2}}{2} \partial_{4}\right)^{(\mathrm{j})} \mathbb{1}_{E}=x_{1}\left(u^{+}-u^{-}\right) a\left(\mathcal{H}^{1}\llcorner J)+\frac{x_{1}^{2}}{2}\left(u^{+}-u^{-}\right) b\left(\mathcal{H}^{1}\llcorner J) .\right.\right.
$$

Altogether, summing the two expressions in (3.28) and (3.29), we get the expression for the $X_{2}$-derivative of $\mathbb{1}_{E}$. The condition of $X_{2}$-monotonicity, i.e.,

$$
\left[\left(\partial_{2}+x_{1} \partial_{3}+\frac{x_{1}^{2}}{2} \partial_{4}\right) \mathbb{1}_{E}\right](h) \geq 0,
$$

for any $h \geq 0$ and for any $x_{1}$, is fulfilled if and only if, ${ }^{2}$ for any $h \geq 0$, the polynomial in $x_{1}$

$$
\int h d \mathcal{L}^{2}-x_{1}\left\langle\left(\partial_{3} G\right)\left(x_{3}, x_{4}\right), h\right\rangle-\frac{x_{1}^{2}}{2}\left\langle\left(\partial_{4} G\right)\left(x_{3}, x_{4}\right), h\right\rangle
$$

is always positive and

$$
\left(x_{1} a+\frac{x_{1}^{2}}{2} b\right) \geq 0 .
$$

The first is in turn equivalent, since such a polynomial has value 1 for $x_{1}=0$, to the discriminant $\left(\partial_{3} G(h)\right)^{2}+2 \partial_{4} G(h)\left\langle\mathcal{L}^{2}, h\right\rangle$ being non-positive.

The second is satisfied if and only if $a=0, b \geq 0$. The vector $(a, b)$ is, on the other hand, the normal $\nu$ to the jump set $J \subset \mathbb{R}^{2}$ of $G$ : so the $X_{2}$-monotonicity is equivalent to $J$ being a countably $\mathcal{H}^{1}$-rectifiable set with constant normal in the direction $x_{4}$, as in the assumptions.

We give now some explicit examples of sets having locally finite horizontal perimeter and constant horizontal normal equal to $X_{2}$ in our model of the Engel group. The first one is a generalization of Example 3.8.

\footnotetext{
${ }^{2}$ Indeed the two measures in (3.28) and (3.29) are mutually singular and the inequality $\left[\left(\partial_{2}+x_{1} \partial_{3}+\frac{x_{1}^{2}}{2} \partial_{4}\right) \mathbb{1}_{E}\right](h)$ splits in the two corresponding inequalities for the two measures $\left(\partial_{2}+x_{1} \partial_{3}+\frac{x_{1}^{2}}{2} \partial_{4}\right)^{\text {cont }} \mathbb{1}_{E}$ and $\left(\partial_{2}+x_{1} \partial_{3}+\frac{x_{1}^{2}}{2} \partial_{4}\right)^{(\mathrm{j})} \mathbb{1}_{E}$. This is proved using bump functions as done in the proof of Lemma 3.22.
} 
Example 3.30. Let $g: \mathbb{R} \rightarrow \mathbb{R}$ be a non-increasing and upper semicontinuous function. Take $K \in(0, \infty)$ and a non-decreasing function $f: \mathbb{R} \rightarrow \mathbb{R}$ Lipschitz continuous with Lipschitz constant $\leq \frac{2}{K^{2}}$. The set

$$
E:=\left\{x \in \mathbb{R}^{4}: x_{2}>f\left(K x_{3}-x_{4}\right)+g\left(x_{4}\right)\right\}
$$

is $X_{2}$-calibrated. We can easily see this fact as a consequence of Theorem 3.17 by computing

$$
\begin{aligned}
\left(\left(\partial_{3} G\right)\left(x_{3}, x_{4}\right)\right)^{2} & =K^{2}\left(f^{\prime}\left(K x_{3}-x_{4}\right)\right)^{2}, \\
\left(\partial_{4} G\right)\left(x_{3}, x_{4}\right) & =-f^{\prime}\left(K x_{3}-x_{4}\right)+\partial_{4} g\left(x_{4}\right)
\end{aligned}
$$

so that

$$
\begin{aligned}
& \left(\left(\partial_{3} G\right)\left(x_{3}, x_{4}\right)\right)^{2}+2\left(\partial_{4} G\right)\left(x_{3}, x_{4}\right) \\
& \quad=\left(K^{2} f^{\prime}\left(K x_{3}-x_{4}\right)-2\right) f^{\prime}\left(K x_{3}-x_{4}\right)+2 \partial_{4} g\left(x_{4}\right) \\
& \quad \leq 2 \partial_{4} g\left(x_{4}\right) \leq 0
\end{aligned}
$$

by the condition on the Lipschitz constant of $f$ and by the monotonicity of $g$.

Example 3.31. The set

$$
C:=\left\{x \in \mathbb{R}^{4}: x_{2}>0, x_{4}>0, x_{2}>\frac{x_{3}^{2}}{2 x_{4}}\right\}
$$

is $X_{2}$-calibrated. In this case we have $G=+\infty$ for $x_{4} \leq 0$ and $G=\frac{x_{3}^{2}}{2 x_{4}}$ for $x_{4}>0$.

Again, making use of Theorem 3.17, we can compute, for $x_{4}>0$ :

$$
\left(\left(\partial_{3} G\right)\left(x_{3}, x_{4}\right)\right)^{2}+2\left(\partial_{4} G\right)\left(x_{3}, x_{4}\right)=\frac{x_{3}^{2}}{x_{4}^{2}}-\frac{x_{3}^{2}}{x_{4}^{2}}=0 .
$$

We remark here that we get 0 because $C$ is a sort of "extreme case", in the sense that, taken any $X_{2}$-calibrated set $E$, if $p \in \partial E$ then we shall prove that $p+C$ must lie in the interior of $E$. This fact will be discussed in detail and play an important role in a subsequent work.

Let us prove the previous claim. Assume that $E$ is $X_{1}$-invariant and $X_{2}$-monotone and let $p=\left(p_{1}, p_{2}, p_{3}, p_{4}\right) \in E$. Then the whole line $\ell=\left\{\left(p_{1}+\right.\right.$ $\left.\left.a, p_{2}, p_{3}, p_{4}\right): a \in \mathbb{R}\right\}$, belongs to $E$. Now the $X_{2}$-monotonicity means that we can flow from any point in $\ell$ for positive times $t$ along $X_{2}$ and we remain 
in $E$. Writing this down explicitly we get

$$
\left(p_{1}+a, p_{2}, p_{3}, p_{4}\right)+\left(0, t,\left(p_{1}+a\right) t, \frac{\left(p_{1}+a\right)^{2}}{2} t\right) \in E \quad \text { for any } t \geq 0, a \in \mathbb{R} .
$$

By using $X_{1}$-invariance again we get that

$$
\left(p_{1}, p_{2}, p_{3}, p_{4}\right)+\left(0, t,\left(p_{1}+a\right) t, \frac{\left(p_{1}+a\right)^{2}}{2} t\right) \in E \quad \text { for any } t \geq 0, a \in \mathbb{R}
$$

The points $\left(t,\left(p_{1}+a\right) t, \frac{\left(p_{1}+a\right)^{2}}{2} t\right)$ with $t \geq 0$ and $a \in \mathbb{R}$ describe the surface $\left\{2 x z=y^{2}: x>0, z>0\right\}$ in $\mathbb{R}^{3}$. This means that whenever $E$ contains $p$ then it must contain the surface $p+\left\{\left(x_{1}, x_{2}, x_{3}, x_{4}\right): x_{2}=\frac{x_{3}^{2}}{2 x_{4}}, x_{2}>0, x_{4}>\right.$ $0\}$. However, recalling that $E$ is an upper-graph in the direction of the $x_{2}$-coordinate we get that $E$ contains the whole $p+\left\{\left(x_{1}, x_{2}, x_{3}, x_{4}\right): x_{2} \geq\right.$ $\left.\frac{x_{3}^{2}}{2 x_{4}}, x_{2}>0, x_{4}>0\right\}$, which is exactly $p+C$. QED

Remark 3.32. The same argument of the previous example shows that if $E$ is a $X_{2}$-calibrated set and $p \in \partial E$ then the $p \cdot C$ must lie in the interior of $E$. Indeed, also the set $p^{-1} \cdot E$ is a $X_{2}$-calibrated set. By the argument above, we have that $C \subseteq p^{-1} \cdot E$ and we are done. Note that $p \cdot C$ is the left translation (with respect to the group structure) of $E$ at $p$. Also note that, since we are in exponential coordinates, we have $C^{-1}=-C$. In addition, if $E$ is a $X_{2}$-calibrated set, then the set $\mathbb{R}^{4} \backslash(-E)$ is $X_{2}$-calibrated. Therefore, for $p \in \partial E$, we have the $p \cdot(-C)$ lies in the interior of the complement of $E$.

\section{Sets with constant horizontal normal as intrinsic horizontal upper-graphs}

In this section we look at the expression of $E$ (a set with constant normal $X)$ as upper-graph of a function when we use as "graphing direction" the flow lines of an horizontal vector field, i.e., we want to describe $E$ as intrinsic horizontal upper-graph in the sense of Definition 1.7 and study the regularity of the graphing function $T$. A natural choice is to use the flow of $X$ as "graphing direction". As we are about to explain in the next subsection, this choice might lead to a surprising lack of regularity for $T$.

Recall that, in view of Lemma 2.2, the only interesting case is when the normal is not $X_{1}$. Moreover, by Lemma 2.3, we can in that case assume without loss of generality that the normal is $X_{2}$. 


\subsection{Intrinsic horizontal upper-graphs in the direction of the normal}

Let $W \subseteq \mathbb{R}^{4}$ be the set of points with second component equal to zero,

$$
W:=\left\{p \in \mathbb{R}^{4}: p_{2}=0\right\}
$$

One can show that $W$ is a subgroup of $\mathbb{R}^{4}$ with respect to the Engel structure given by (3.1). Indeed, to see this, it is enough to observe the following two facts. First, the vector space spanned by the vector fields $X_{1}, X_{3}, X_{4}$ form a Lie sub-algebra. The second fact to notice is that the span of such vectors is tangent to $W$. Thus $W$ is a subgroup whose Lie algebra has basis $X_{1}, X_{3}, X_{4}$. From the algebraic viewpoint, the subgroup $W$ is a complementary subgroup of the one-parameter subgroup tangent to the vector field $X_{2}$. From the geometric viewpoint, for each $p \in \mathbb{R}^{4}$, the three-dimensional plane $W$ intersects the line $t \mapsto p \exp \left(t X_{2}\right)$ in one and only one point. Indeed, by (3.6) the second coordinate of $p \exp \left(t X_{2}\right)$ is $p_{2}+t$, which is zero when (and only when) $t=-p_{2}$. We conclude that the space $\mathbb{R}^{4}$ can be parameterized by the following map

$$
\begin{gathered}
\Psi: W \times \mathbb{R} \rightarrow \mathbb{R}^{4} \\
(p, t) \mapsto p \exp \left(t X_{2}\right) .
\end{gathered}
$$

Assume now that $E \subseteq \mathbb{R}^{4}$ is a $X_{2}$-calibrated set. We plan to write $E$ as an upper-graph of a function. By the definition of $X_{2}$-calibration there exists a map $p \mapsto T(p)$ from $\mathbb{R}^{4}$ to $\overline{\mathbb{R}}$ such that

$$
\left.\left\{t: p \exp \left(t X_{2}\right)\right) \in E\right\}=(T(p),+\infty)
$$

Restricting such a map $T$ to $W$. We get that

$$
\begin{aligned}
E & =\{\Psi(p, t): p \in W, t>T(p)\} \\
& =\{\Psi(p, T(p)+t): p \in W, t>0\} . \\
& =\left\{\left(p_{1}, t, p_{3}+p_{1} t, p_{4}+p_{1}^{2} t / 2\right): p \in W, t>T(p)\right\} .
\end{aligned}
$$

Let us study the map $p \mapsto T(p)$ from $W$ to $\mathbb{R}$, in the examples Example 3.8 where $E:=\left\{x \in \mathbb{R}^{4}: x_{2}+g\left(x_{4}\right)>0\right\}$, with $g$ non-decreasing and upper semicontinuous. The value $T(p)$ is the lower value $T$ such that

$$
T+g\left(p_{4}+p_{1}^{2} T\right) \geq 0,
$$


since on $W$ we have $p_{2}=0$. Restrict the map $T$ to $W \cap\left\{p_{1}=0\right\}$, so

$$
T(p)=-g\left(p_{4}\right)
$$

In conclusion, $T: W \rightarrow \mathbb{R}$ is as much non-regular as $g$ is. In particular, there are examples of non-continuous function $T$. We can summarize the last discussion in the following fact.

Theorem 4.1. For any $X \neq X_{1}$ there are examples of sets $E$ with constant horizontal normal $X$ and with the property that, when they are written as intrinsic horizontal upper-graphs in the direction of $X$, the function for which they are upper-graphs is not continuous.

\subsection{Graphs in other horizontal directions}

For a set $E$ with constant normal $X_{2}$, the previous example has shown that there can be a lack of continuity for the intrinsic graph representing the boundary of $E$ when we choose the flow lines of $X_{2}$ as graphing directions.

We might however still look at what happens when the boundary of $E$ is represented as an intrinsic graph using different horizontal flow lines as graphing direction: namely let us observe the flow lines of $a X_{1}+X_{2}$ for $a>0$.

First of all we need to write down, analogously to what was done in (3.5), the flow of $a X_{1}+X_{2}$ in the model of the Engel group considered so far. What we get is that the flow line starting at $\left(p_{1}, p_{2}, p_{3}, p_{4}\right)$ is

$$
\Phi(p, t):=\left(p_{1}+a t, p_{2}+t, p_{3}+p_{1} t+\frac{a}{2} t^{2}, p_{4}+\frac{p_{1}^{2}}{2} t+\frac{a p_{1}}{2} t^{2}+\frac{a^{2}}{6} t^{3}\right) .
$$

We are going to show now that this intrinsic graph might fail to be Euclidean Lipschitz. To see this, we will consider the set in Example 3.31.

Theorem 4.3. When the set $C:=\left\{x \in \mathbb{R}^{4}: x_{2}>0, x_{4}>0, x_{2}>\frac{x_{3}^{2}}{2 x_{4}}\right\}$ is represented as intrinsic upper-graph in any horizontal direction $a X_{1}+X_{2}$, the function for which it is upper-graph is not Euclidean Lipschitz.

Proof of Theorem 4.3. The intrinsic function $T(p)$ yielding the upper-graph is a function $T: W \rightarrow \mathbb{R}$, where $W:=\left\{p \in \mathbb{R}^{4}: p_{2}=0\right\}$ as before. The value $T(p)$ is the infimum of the times $t$ for which the flow line starting at $p$ is inside $C$. 
Let us restrict the attention to points in $W$ with $p_{1}=p_{3}=0$. The flow lines are then $\left(a t, t, \frac{a}{2} t^{2}, p_{4}+\frac{a^{2}}{6} t^{3}\right)$ and we must see when the flow line enters the set $C$. The value $T(p)$ for $p_{4} \geq 0$ is clearly 0 , while for $p_{4}<0$ it is the solution of the following equation in $t$

$$
2 t\left(p_{4}+\frac{a^{2}}{6} t^{3}\right)=\frac{a^{2}}{4} t^{4},
$$

with the constraint that $p_{4}+\frac{a^{2}}{6} t^{3}>0$.

Solving this equation we get $\frac{a^{2}}{12} t^{3}=2\left|p_{4}\right|$, i.e., $t=\sqrt[3]{\frac{24\left|p_{4}\right|}{a^{2}}}$, which fulfils the constraint $p_{4}+\frac{a^{2}}{6} t^{3}>0$. So we have that, restricting to $p_{1}=p_{3}=0$ in $W$, the function for which $C$ is upper-graph is $\sqrt[3]{\frac{24\left|p_{4}\right|}{a^{2}}}$ for $p_{4}<0$ and identically 0 for $p_{4} \geq 0$, so it is not Lipschitz continuous for the Euclidean distance.

It is therefore necessary to use non-horizontal directions as "graphing direction" (as done in Corollary 3.14) in order to see the Euclidean Lipschitz continuity of the function describing the boundary of a set with constant horizontal normal.

The previous proof leaves however still open the possibility for the intrinsic graph in the direction $a X_{1}+X_{2}$ to be Lipschitz with respect to the intrinsic subRiemannian geometry of $\mathbb{G}$, as we are about to discuss. In particular, we shall obtain Hölder continuity of the graphing function with respect to the Euclidean distance.

Let us recall the notion of intrinsic cones and intrinsic Lipschitz regularity for graphs in Carnot groups. Given a group $\mathbb{G}$ and two subgroups $W, H \subseteq \mathbb{G}$, we say that $W$ and $H$ are two complementary subgroups if $\mathbb{G}=W \cdot H$ and $W \cap H=\{1\}$. Given a function $f: W \rightarrow H$ between two subgroups, its graph is the set

$$
\Gamma_{f}:=\{w \cdot f(w): w \in W\} \subset \mathbb{G}
$$

Definition 4.4 (Intrinsic cones). Let $\mathbb{G}$ be a group admitting dilations $\delta_{\lambda}$, for all $\lambda \in \mathbb{R}$. Let $H \subseteq \mathbb{G}$ be a subgroup of $\mathbb{G}$. An open set $C \subset \mathbb{G}$ is called intrinsic open cone with axis $H$ if $C=\delta_{\lambda}(C)$, for all $\lambda \in \mathbb{R}$, and $H \subseteq$ $\{1\} \cup C$.

Definition 4.5 (Intrinsic Lipschitz graphs). Let $W, H \subseteq \mathbb{G}$ be two complementary subgroups of a group $\mathbb{G}$ admitting dilations. Then a function $f: W \rightarrow H$ is called intrinsic Lipschitz (or Lipschitz in the sense of 
Franchi-Serapioni-Serra Cassano) if there exists an intrinsic open cone with axis $H$ such that

$$
\Gamma_{f} \cap(p \cdot C)=\emptyset, \quad \forall p \in \Gamma_{f}
$$

The above definition has been introduced by Franchi et al., see [15, 16]. We phrased the definition a little bit differently, however, it is easy to see that it is equivalent.

Theorem 4.6. When a set $E$ with constant horizontal normal $X_{2}$ is represented as intrinsic horizontal upper-graph in any horizontal direction $a X_{1}+$ $X_{2}$, with $a \neq 0$, the function for which it is upper-graph is intrinsically Lipschitz-continuous.

Proof of Theorem 4.6. In order to prove the theorem, we show the intrinsic cone in the coordinates given by (3.1):

$$
\tilde{C}:=\left\{2 x_{2} x_{4}>x_{3}^{2}\right\}
$$

It is clear that $\tilde{C}$ is open and invariant under the group dilations:

$$
\left(x_{1}, x_{2}, x_{3}, x_{4}\right) \mapsto\left(\lambda x_{1}, \lambda x_{2}, \lambda^{2} x_{3}, \lambda^{3} x_{4}\right) .
$$

By (4.2), we have that

$$
\exp \left(t\left(a X_{1}+X_{2}\right)\right)=\Phi(0, t)=\left(a t, t, \frac{a}{2} t^{2}, \frac{a^{2}}{6} t^{3}\right) .
$$

If $t \neq 0$, then such a point is in $\tilde{C}$, since

$$
2 \Phi_{2}(0, t) \Phi_{4}(0, t)=2 t \frac{a^{2}}{6} t^{3}=\frac{a^{2}}{3} t^{4}>\frac{a^{2}}{4} t^{4}=\left(\frac{a}{2} t^{2}\right)^{2}=\Phi_{3}(0, t)^{2} .
$$

Hence, if $H:=\exp \left(\mathbb{R}\left(a X_{1}+X_{2}\right)\right)$, we have $H \subseteq\{1\} \cup \tilde{C}$. So $\tilde{C}$ is an intrinsic open cone with axis $H$.

It is important that we notice that $\tilde{C}$ is the cone obtained as the previous set $C$ of Example 3.31 together with its reflection:

$$
\tilde{C}=C \cup(-C)=C \cup C^{-1} .
$$

Let $E$ be a set with normal $X_{2}$. Up to replacing it by its Lebesgue representative (see Section 2.2), we may assume that $E$ is $X_{2}$-calibrated (see Definition 3.7). By Remark 3.32, we know that if $p \in \partial E$, then $p \cdot C \subseteq E$ 
and similarly $p \cdot\left(C^{-1}\right) \subseteq \mathbb{R}^{4} \backslash E$. Now let $W$ be, as before, the set $\left\{p \in \mathbb{R}^{4}\right.$ : $\left.p_{2}=0\right\}$. Let $f: W \rightarrow H$ be the function for which $\Gamma_{f}=\partial E$. Then, if $p \in \Gamma_{f}$, we have $\Gamma_{f} \cap(p \cdot C)=\emptyset$.

The above proof showed that the cone $C$ has the property of containing the direction $a X_{1}+X_{2}$ in its interior, when $a \neq 0$. Since such a cone is an intrinsic cone, hence invariant under inhomogeneous dilations, we can find a cusp of the form

$$
Q:=\left\{x \in \mathbb{R}^{4}:\langle x, v\rangle \in(0,1), \operatorname{dist}(x, \mathbb{R} v)<K(\langle x, v\rangle)^{3}\right\}
$$

for some $K>0$ and $v=a e_{1}+e_{2}$, contained in $C$. Since the intrinsic graph in the direction $a X_{1}+X_{2}$ avoid the cusp, we can conclude that it is Hölder. Hence, we showed the following corollary.

Lemma 4.7. When a $X_{2}$-calibrated set $E$ is represented as intrinsic uppergraph in any horizontal direction $a X_{1}+X_{2}$, with $a \neq 0$, the function for which it is upper-graph is locally Euclidean Hölder-continuous.

We remark that the fact that intrinsic Lipschitz graphs are locally Hölder-continuous holds in complete generality in any stratified group. One can easily generalize our geometric reasoning given above. A more analytic proof will be contained in [13].

\section{Regularity in filiform groups}

A stratified group $\mathbb{G}$ is said to be a filiform group if the strata $V_{j}$ of the stratification

$$
\operatorname{Lie}(\mathbb{G})=V_{1} \oplus \cdots \oplus V_{s}
$$

of the Lie algebra $\operatorname{Lie}(\mathbb{G})$ are such that $\operatorname{dim} V_{1}=2$ and $\operatorname{dim} V_{j}=1$, for $j=2, \ldots, s$. Here $s$ is the step of the group.

One can easily show that there exists a basis $X_{0}, \ldots, X_{s}$ of $\operatorname{Lie}(\mathbb{G})$ by vectors with the following property: $X_{0}, X_{1} \in V_{1}, X_{j} \in V_{j}$, for $j=2, \ldots, s$, and

$$
\left[X_{0}, X_{j-1}\right]=X_{j}, \quad \text { for } j=2, \ldots, s
$$

In general there might be other non-null brackets of elements of this basis. In [24], Vergne gave a classification of all stratified filiform groups. In fact, in [24, Corollary 1, page 93], Vergne showed that, in the case the step $s$ is 
even (so the dimension of the group is odd), then there is only one stratified group of step $s$ and it admits a basis for which the brackets are all null, except those in (5.1). In case $s$ is odd (and the dimension is even), then there are only two different filiform groups: one where, a part from (5.1), all other brackets are null and a second one where the only other non-null bracket relation is

$$
\left[X_{l}, X_{s-l}\right]=(-1)^{l} X_{s}, \quad \text { for } l=1, \ldots, s-1
$$

We refer to this two groups as the filiform group of the first kind and the filiform group of the second kind, respectively.

We shall show how the argument for proving the regularity of constantnormal sets in the Engel group can be extended to any filiform group of the first kind.

Theorem 5.2. Let $\mathbb{G}$ be any filiform group of the first kind. Let $E \subset \mathbb{G}$ be a set with horizontal constant normal. Then $E$ is a Lipschitz domain.

Proof. Let $X_{0}, \ldots X_{s}$ be a basis of Lie(G) satisfying (5.1). As for the Engel group, we can assume that either $X_{0} \mathbb{1}_{E}=0$ or $X_{1} \mathbb{1}_{E}=0$. Consider first the case $X_{0} \mathbb{1}_{E}=0$. By Proposition 2.1, the vector $\operatorname{Ad}_{\exp \left(t X_{0}\right)} X_{1}$ is a monotone direction. Explicitly, by (5.1), we have

$$
\operatorname{Ad}_{\exp \left(t X_{0}\right)} X_{1}=e^{\operatorname{ad}\left(t X_{0}\right)} X_{1}=X_{1}+t X_{2}+\frac{t^{2}}{2} X_{3}+\cdots+\frac{t^{s-1}}{s-1} X_{s}
$$

Pick $s$ distinct numbers $t_{1}, \ldots, t_{s}$. Consider the vectors

$$
Y_{j}=\operatorname{Ad}_{\exp \left(t_{j} X_{0}\right)} X_{1}, \quad \text { for } j=1, \ldots, s .
$$

We claim that the vectors $Y_{j}$ are linearly independent. Indeed, it is enough to show that the matrix

$$
\left(\begin{array}{ccccc}
1 & t_{1} & \frac{t_{1}^{2}}{2} & \ldots & \frac{t_{1}^{s-1}}{s-1} \\
\vdots & \vdots & \vdots & \ddots & \vdots \\
1 & t_{s} & \frac{t_{s}^{2}}{2} & \ldots & \frac{t_{s}^{s-1}}{s-1}
\end{array}\right)
$$


has full rank. Equivalently, we need

$$
\operatorname{det}\left(\begin{array}{ccccc}
1 & t_{1} & t_{1}^{2} & \ldots & t_{1}^{s-1} \\
\vdots & \vdots & \vdots & \ddots & \vdots \\
1 & t_{s} & t_{s}^{2} & \ldots & t_{s}^{s-1}
\end{array}\right) \neq 0
$$

We observe that we are considering a Vandermonde Matrix. Hence such a determinant is $\Pi_{1 \leq i<j<s}\left(t_{i}-t_{j}\right)$, which is non-zero, since the $t_{j}$ 's have been chosen to be distinct. Since we found a basis of monotone directions, as for the Engel group, we conclude that the set $E$ is (equivalent) to a Lipschitz domain.

Let us consider now the case $X_{1} \mathbb{1}_{E}=0$. Applying Proposition 2.1, we get that the vector field

$$
\operatorname{Ad}_{\exp \left(t X_{1}\right)} X_{0}=X_{0}-t X_{2}
$$

is a monotone direction, for all $t \in \mathbb{R}$. Thus $X_{2} \mathbb{1}_{E}=0$. Iterating the use of Proposition 2.1 and using (5.1), we get that all vectors $X_{2}, \ldots, X_{s}$ are invariant directions. Hence, $E$ is half-space.

\section{Acknowledgments}

Both authors would like to acknowledge ETH Zürich for its supporting research environment while part of this work was conducted. This paper has benefited from numerous discussions with Luigi Ambrosio, Bruce Kleiner, Raul Serapioni, Francesco Serra Cassano, and Davide Vittone and from the anonymous reports of referees. Special thanks go to them. The first author acknowledges financial support from the Giorgio and Elena Petronio Fellowship and the National Science Foundation under agreement No. DMS-1128155.

\section{References}

[1] L. Ambrosio, N. Fusco and D. Pallara, Functions of bounded variation and free discontinuity problems, Oxford Mathematical Monographs, The Clarendon Press, Oxford University Press, New York, 2000.

[2] L. Ambrosio, B. Kleiner and E. Le Donne, Rectifiability of sets of finite perimeter in Carnot groups: existence of a tangent hyperplane, J. Geom. Anal. 19(3) (2009), 509-540. 
[3] L. Ambrosio, F. S. Cassano and D. Vittone, Intrinsic regular hypersurfaces in Heisenberg groups, J. Geom. Anal. 16(2) (2006), 187-232.

[4] V. B. Adesi, F. S. Cassano and D. Vittone, The Bernstein problem for intrinsic graphs in Heisenberg groups and calibrations, Calc. Var. Partial Different. Equ. 30(1) (2007), 17-49.

[5] F. Bigolin and F. S. Cassano, Distributional solutions of Burgers' equation and intrinsic regular graphs in Heisenberg groups, J. Math. Anal. Appl. 366(2) (2010), 561-568.

[6] F. Bigolin and Francesco Serra Cassano, Intrinsic regular graphs in Heisenberg groups vs. weak solutions of non-linear first-order PDEs, Adv. Calc. Var. 3(1) (2010), 69-97.

[7] F. Bigolin and D. Vittone, Some remarks about parametrizations of intrinsic regular surfaces in the Heisenberg group, Publ. Mat. 54(1) (2010), 159-172.

[8] L. Capogna, D. Danielli, S.D. Pauls, J.T. Tyson, An introduction to the Heisenberg group and the sub-Riemannian isoperimetric problem, Progress in Mathematics, vol. 259, Birkhäuser-Verlag, Basel, 2007.

[9] J. Cheeger and B. Kleiner, Differentiating maps into $L^{1}$, and the geometry of BV functions, Ann. Math. (2) 171(2) (2010), 1347-1385.

[10] E. De Giorgi, Su una teoria generale della misura $(r-1)$-dimensionale in uno spazio ad $r$ dimensioni, Ann. Mat. Pura Appl. (4) 36 (1954), 191-213.

[11] _ Nuovi teoremi relativi alle misure $(r-1)$-dimensionali in uno spazio ad $r$ dimensioni, Ricerche Mat. 4 (1955), 95-113.

[12] _ Frontiere orientate di misura minima, Seminario di Matematica della Scuola Normale Superiore di Pisa, 1960-61, Editrice Tecnico Scientifica, Pisa, 1961.

[13] B. Franchi, M. Marchi and R. Serapioni, Intrinsic Lipschitz functions and a Rademacher type theorem, Manuscript (2012).

[14] B. Franchi, R. Serapioni and F. S. Cassano, On the structure of finite perimeter sets in step 2 Carnot groups, J. Geom. Anal. 13(3) (2003), 421-466.

[15] _ Intrinsic Lipschitz graphs in Heisenberg groups, J. Nonlinear Convex Anal. 7(3) (2006), 423-441. 
[16] _ Differentiability of intrinsic Lipschitz functions within Heisenberg groups, J. Geom. Anal. 21(4) (2011), 1044-1084.

[17] E. Giusti, Minimal surfaces and functions of bounded variation, Monographs in Mathematics, vol. 80, Birkhäuser Verlag, Basel, 1984.

[18] M. Giaquinta, G. Modica and J. Souček, Cartesian currents in the calculus of variations. I, Ergebnisse der Mathematik und ihrer Grenzgebiete. 3. Folge. A Series of Modern Surveys in Mathematics [Results in Mathematics and Related Areas. 3rd Series. A Series of Modern Surveys in Mathematics], vol. 37, Springer-Verlag, Berlin, 1998, Cartesian currents.

[19] B. Kirchheim and F. S. Cassano, Rectifiability and parameterization of intrinsic regular surfaces in the Heisenberg group, Ann. Sc. Norm. Super. Pisa Cl. Sci. (5) 3(4) (2004), 871-896.

[20] Enrico Le Donne, Lecture notes on sub-Riemannian geometry, Preprint available on https://sites.google.com/site/enricoledonne/ (2010).

[21] M. Miranda, Superficie minime illimitate, Ann. Scuola Norm. Sup. Pisa Cl. Sci. (4) 4(2) (1977), 313-322.

[22] R. Monti, Francesco Serra Cassano and D. Vittone, A negative answer to the Bernstein problem for intrinsic graphs in the Heisenberg group, Boll. Unione Mat. Ital. (9) 1(3) (2008), 709-727.

[23] R. Monti and D. Vittone, Sets with finite $\mathbb{H}$-perimeter and controlled normal, Mathematische Zeitschrift (2011), 1-17, doi: 10.1007/s00209010-0801-7.

[24] M. Vergne, Cohomologie des algèbres de Lie nilpotentes. Application à l'étude de la variété des algèbres de Lie nilpotentes, Bull. Soc. Math. France 98 (1970), 81-116.

[25] D. Vittone, Submanifolds in Carnot groups, Tesi. Scuola Normale Superiore di Pisa (Nuova Series) [Theses of Scuola Normale Superiore di Pisa (New Series)], vol. 7, Edizioni della Normale, Pisa, 2008, Thesis, Scuola Normale Superiore, Pisa, 2008. 
Princeton University

Fine HaLL

WASHINGTON ROAD

PRINCETON 08544-1000

NJ,USA

Institute for Advanced Study

EINSTEIN DRIVE

PRINCETON 08540

NJ, USA

Department of Mathematics and Statistics

UNIVERSITY OF JYVÄSKYLÄ

40014 JYVÄSKYL ̈̈

FINLAND

E-mail address: cbellett@math.princeton.edu

E-mail address: costante@math.ias.edu

E-mail address: ledonne@msri.org

ReCeIVed March 26, 2012 
